OPEN ACCESS

Edited by:

Charles McMaster Oman, Massachusetts Institute of

Technology, United States

Reviewed by:

Robert V. Kenyon,

University of Illinois at Chicago,

United States

Séamas Weech,

McGill University, Canada

*Correspondence:

Stephen Palmisano

stephenp@uow.edu.au

Specialty section:

This article was submitted to Virtual Reality and Human Behaviour,

a section of the journa

Frontiers in Virtual Reality

Received: 27 July 2020 Accepted: 02 October 2020 Published: 12 November 2020

Citation:

Palmisano S, Allison RS and Kim J (2020) Cybersickness in Head-Mounted Displays Is Caused by Differences in the User's Virtual and Physical Head Pose.

Front. Virtual Real. 1:587698. doi: 10.3389/frvir.2020.587698

\section{Cybersickness in Head-Mounted Displays Is Caused by Differences in the User's Virtual and Physical Head Pose}

\author{
Stephen Palmisano ${ }^{1 *}$, Robert S. Allison ${ }^{2,3}$ and Juno Kim ${ }^{4}$ \\ ${ }^{1}$ School of Psychology, University of Wollongong, Wollongong, NSW, Australia, ${ }^{2}$ Centre for Vision Research, York University, \\ Toronto, ON, Canada, ${ }^{3}$ Department of Electrical Engineering and Computer Science, York University, Toronto, ON, Canada, \\ ${ }^{4}$ School of Optometry and Vision Science, University of New South Wales, Sydney, NSW, Australia
}

Sensory conflict, eye-movement, and postural instability theories each have difficulty accounting for the motion sickness experienced during head-mounted display based virtual reality (HMD VR). In this paper we review the limitations of existing theories in explaining cybersickness and propose a practical alternative approach. We start by providing a clear operational definition of provocative motion stimulation during active HMD VR. In this situation, whenever the user makes a head movement, his/her virtual head will tend to trail its true position and orientation due to the display lag (or motion to photon latency). Importantly, these differences in virtual and physical head pose (DVP) will vary over time. Based on our own research findings, we propose that cybersickness in HMD VR is triggered by large magnitude, time-varying patterns of DVP. We then show how this hypothesis can be tested by: (1) systematically manipulating display lag magnitudes and head movement speeds across HMD VR conditions; and (2) comparing the estimates of the user's DVP in each of these conditions to their own reports of cybersickness severity. We believe that this approach will allow researchers to precisely predict which situations will (and will not) be provocative for cybersickness in HMD VR.

Keywords: head-mounted display, motion sickness, cybersickness, motion-to-photon latency, sensory conflict, postural instability

\section{INTRODUCTION}

Anyone who has tried virtual reality (VR) using modern head-mounted displays (HMDs) cannot help but be impressed by their potential. These increasingly affordable, consumer-friendly devices are now able to transport their users to highly immersive computer-generated worlds. The interactive, multisensory feedback that they provide can generate compelling feelings of presence (or "being there") and realistic user responses to these virtual environments (Schubert et al., 2001; Cummings and Bailenson, 2016; Skarbez et al., 2017).

The promise of this revolutionary technology can clearly be seen by the host of applications already developed for its use (e.g., in SteamVR, Oculus and Viveport). To date, HMD VR applications have been created for advertising, archaeology, architecture, business, clinical psychology, defense, design, education, engineering, entertainment and the arts, health and safety, gaming, manufacturing, medicine, real estate, research, simulation training, sport, social media, 
telecommunications, tourism, and urban design (e.g., Tate et al., 1997; Hogue et al., 1999; Blascovich et al., 2002; Simons and Melzer, 2003; Mujber et al., 2004; Villani et al., 2007; Ch'ng, 2009; Phan and Choo, 2010; Wiederhold et al., 2014; Gonizzi Barsanti et al., 2015; Grabowski and Jankowski, 2015; Elliman et al., 2016; Eubanks et al., 2016; Khor et al., 2016; Ortegon-Sarmiento et al., 2016; Bernardo, 2017; Andersen and Popescu, 2018; Jensen and Konradsen, 2018; Pot-Kolder et al., 2018; Han and Cho, 2019; Yildirim, 2019a,b; Chen et al., 2020).

Unfortunately, despite the potential of HMD VR, user experiences of motion sickness continue to limit its adoption (Biocca, 1992; Draper et al., 2001; Patterson et al., 2006; Merhi et al., 2007; Sharples et al., 2008; Lawson, 2014; Rebenitsch and Owen, 2016, 2020; Munafo et al., 2017; Palmisano et al., 2017; Weech et al., 2018; Arcioni et al., 2019; Clifton and Palmisano, 2019; Risi and Palmisano, 2019). This paper is focused on better understanding this cybersickness ${ }^{1}$, as well as proposing new ways to study, and potentially mitigate, it.

\section{The Problem of Cybersickness}

Despite heavy investment in possible hardware and software solutions over the last decade, many users still become sick during HMD VR (Rebenitsch and Owen, 2016, 2020). For example, in our recent research using modern HMDs and commercial video games, more than $80 \%$ of participants reported some cybersickness after only 10-15 min of HMD VR gameplay (Clifton and Palmisano, 2019; Risi and Palmisano, 2019; Teixeira and Palmisano, 2020). From their own anecdotal reports, these HMD users appear to transition quite rapidly from pleasurable feelings of immersion to unpleasant experiences of cybersickness (see Boyd, 2014; Lewis, 2015). This cybersickness can present as a variety of signs and symptoms, including nausea, stomach awareness, increased/decreased salivation, sensations of bodily warmth, sweating, changes in facial pallor, disorientation, dizziness, vertigo, fainting, light headedness, fullness of head, blurred vision, eye strain, difficulty focusing, drowsiness, headache, fatigue, and sometimes even vomiting and retching (Ebenholtz, 1992; McCauley and Sharkey, 1992; Stanney et al., 1998b; LaViola, 2000; Lawson, 2014; Rebenitsch and Owen, 2016; Gavgani et al., 2017a).

Unfortunately, cybersickness in HMD VR tends to be more provocative than the sickness produced by other types of VR (Howarth and Costello, 1997; Sharples et al., 2008; Kim et al., 2014; Dennison et al., 2016; Yildirim, 2019a,b). For example, in a recent study, Dennison et al. (2016) found that while 11 of their 20 participants were too sick to continue the HMD VR simulation, none of them dropped out when the same simulation was presented via desktop VR. Similarly, Yildirim (2019a) found that cybersickness was common after only 6 min of HMD VR gameplay, whereas minimal sickness was produced when playing desktop versions of the same games.

\footnotetext{
${ }^{1}$ Cybersickness refers to sickness experienced in both HMD and non-HMD VR. It can also be used to describe the adverse effects produced by large projection/dome screens, Cave Automatic Virtual Environments (CAVE), and VR theaters (McCauley and Sharkey, 1992).
}

If this cybersickness cannot be substantially reduced, then HMD gaming may fail commercially (as 3D television did recently for home entertainment). We therefore need a better understanding of both the causes and the development of cybersickness in HMD VR, so that we can find more effective ways to mitigate it. While the experience of cybersickness can vary substantially from one HMD user to another (McCauley and Sharkey, 1992): (1) disorientation appears to be a very common symptom (Lawson, 2014; Rebenitsch and Owen, 2016); and (2) vomiting during or after HMD VR is rare (Stanney et al., 1998b; Kingdon et al., 2001). According to Kennedy et al. (2010), HMD VR also tends to cause more nausea, and fewer oculomotor, symptoms than non-HMD VR. These cybersickness symptoms can persist even after the user removes their HMD. In some cases, they can still be reported up to $12 \mathrm{~h}$ after the exposure (Kennedy and Lilienthal, 1994; Kennedy et al., 1994; Merhi et al., 2007).

Lawson (2014) has recently noted that there is "no comprehensive and universally accepted theory of motion sickness etiology" (p. 533). This statement also applies to the cybersickness experienced in HMD VR. Thus, in this paper, we propose a new way to understand and study this cybersickness. However, before we outline our hypothesis and recommend an approach for testing it, we will first review the existing theories of cybersickness and their supporting evidence.

\section{PART 1: REVIEW OF EXISTING THEORIES OF CYBERSICKNESS}

Most current theories of cybersickness were originally created to explain motion sickness in the real world (such as car, sea, and air sickness) or in vehicle simulators. A variety of different triggers have been proposed for this sickness, including sensory conflict, neural mismatch, visual illusions of self-motion, errors in perceiving the direction of gravity or which parts of the scene are stationary, increased postural instability, excessive eye-motion, and even misperceptions of poisoning (Reason and Brand, 1975; Treisman, 1977; Reason, 1978; Oman, 1982, 1990; Hettinger et al., 1990; Riccio and Stoffregen, 1991; Ebenholtz, 1992; Ebenholtz et al., 1994; Bles et al., 1998; Stoffregen and Smart, 1998; Nalivaiko et al., 2014). Below we outline these different explanations of motion sickness and discuss the evidence for and against them (including data in HMD VR where it is available).

\section{Sensory Conflict Theories of Motion Sickness}

Sensory conflict remains the most cited explanation for all types of motion sickness, including cybersickness (Keshavarz et al., 2014; Rebenitsch and Owen, 2016; Yildirim, 2019a). These theories focus on the self-motion and orientation information provided by vision, the vestibular system of the inner ear and the other non-vestibular proprioceptive senses (Guedry, 1991; Palmisano et al., 2011a,b; Keshavarz et al., 2014). Each of these sensory systems has its own specializations and limitations. For example, while vision can detect a variety of self-motions (based on the optic flow that we see when we move), the vestibular 
system is specialized for detecting accelerating head motion and orientation with respect to gravity (with the semicircular canals and otolith organs responding best to angular and linear head accelerations, respectively; Howard, 1982). Other useful sources of information are also provided about our orientation with respect to gravity based on visual frame and polarity cues, as well as the inertia of our limbs and the forces acting on our bodies (Lishman and Lee, 1973; Lee and Lishman, 1975; Howard, 1982; Howard and Childerson, 1994; Allison et al., 1999; Howard and $\mathrm{Hu}, 2001)$.

It is commonly assumed that motion sickness can be triggered whenever two or more of the above sensory systems provide contradictory information (Claremont, 1931; Reason and Brand, 1975). For example, Hettinger et al. (1990) argued that the motion sickness experienced during visually induced illusions of selfmotion was due to visual-vestibular conflict. In this case, the observer's optic flow indicates that he/she is moving, but the lack of corresponding activity from the inner ears suggests (correctly) that he/she is stationary. However, this is only one of a number of different sensory conflict accounts of motion sickness (see Treisman, 1977; Reason, 1978; Oman, 1982, 1990; Bles et al., 1998; Prothero and Parker, 2003). Below we first describe the best known, and most highly cited, of these sensory conflict theories of motion sickness: the sensory rearrangement theory (Reason, 1978). We then proceed on to describe: (1) the modifications that have been made to this theory over the years; and (2) some alternative hypotheses about the exact relationships between sensory conflict and motion sickness.

\section{Sensory Rearrangement Theory}

According to this theory, sensory conflict alone is not sufficient to induce motion sickness (Reason, 1978). It is assumed that we have access to a neural store of every pattern of motion stimulation that we have ever been exposed to. Whenever we plan a movement, the expected pattern of multisensory stimulation for this movement is chosen from the neural store. After the movement is initiated, this expected pattern is then compared to the actual pattern of stimulation arriving from our senses. According to the theory, motion sickness should only occur when there is a discrepancy between our currently sensed and expected patterns of stimulation, referred to as a neural mismatch. The likelihood of us becoming sick, and the severity of our sickness, should increase with the degree of this neural mismatch. Our motion sickness should also decrease with repeated exposures to an initially provocative stimulus. This is because our neural store will be recalibrated during each exposure, resulting in a little less neural mismatch on each subsequent exposure. While the theory is focused on planned self-motions, it predicts that motion sickness should be even more likely when we are not in control of our motion (e.g., when we are passengers in a moving automobile).

\section{Criticisms of sensory rearrangement theory}

It is generally acknowledged that sensory rearrangement theory can provide convincing post-hoc explanations of the motion sickness findings of many past studies (Rolnick and Lubow, 1991; Howarth and Finch, 1999; Hill and Howarth, 2000; Draper et al.,
2001; Akiduki et al., 2003; Bonato et al., 2005, 2008, 2009; Bubka et al., 2007; Palmisano et al., 2007, 2017; Howarth and Hodder, 2008; Keshavarz and Hecht, 2011a; Nishiike et al., 2013; Chen et al., 2016; Gavgani et al., 2017b). However, it has often been criticized for its inability to make precise, quantitative predictions about motion sickness in the future (Stoffregen and Riccio, 1991; McCauley and Sharkey, 1992; Bles et al., 1998; Draper et al., 2001; Davis et al., 2014; Keshavarz et al., 2014; Lawson, 2014; Lackner and DiZio, 2020). In their recent review, Keshavarz et al. (2014) noted that "the range of conceivable conflicts is so wide that it is difficult to devise experiments [that] would falsify the theory" (p. 654). Lackner and DiZio (2020) have also argued that because "we do not have an adequate understanding of the formation, nature and operation of [the neural store]" (p. 1212) this limits the predictive and explanatory capability of the theory. Researchers attempting to test the theory are forced to make assumptions about: (1) whether a particular stimulus will produce a neural mismatch or not; and (2) if it does, how much mismatch will be generated. The need to make such assumptions clearly limits the practical utility of the theory for studying motion sickness. Thus, as Ebenholtz et al. (1994) note "in its present form, [sensory rearrangement theory] may be untestable" (p. 1034).

\section{Mathematical model of sensory rearrangement theory}

In an attempt to address these criticisms, Oman (1982, 1990) created a mathematical model of sensory rearrangement theory. In this model: (1) muscular activity $(\mathbf{m})$ is generated to move toward a desired destination $\left(\mathbf{x}_{\mathrm{d}}\right),(2)$ due to actual body dynamics (B), this results in movement to position $\mathbf{x}$ at time 1; (3) the consequences of this movement are detected by the senses (S) in the presence of external noise $\left(\mathbf{n}_{\mathrm{e}}\right)$, resulting in a sensory outcome $(\mathbf{a})$; (4) the neural store computes the expected sensory outcome ( $\hat{\mathbf{a}}$ ) of the movement, based on $\mathbf{m}$ and internal estimates of the other components (i.e., $\hat{\mathbf{x}}, \hat{\mathbf{S}}$, and $\hat{\mathbf{B}}$ ); and 5) the motion sickness produced is estimated as the vector difference between these actual (a) and expected (â) sensory outcomes. The greater the vector difference, the more likely the model will be to trigger motion sickness, and the more severe it will be. A weighted amount of this vector difference is also fed back into the model to update the neural store, allowing it to simulate the sensory adaptation/habituation that occurs during repeated exposures to initially provocative stimuli (Hill and Howarth, 2000; Howarth and Hodder, 2008).

While this mathematical model represents a considerable improvement on earlier versions of sensory rearrangement theory (Reason and Brand, 1975; Reason, 1978), assumptions still need to be made about its input parameters, connection weightings, and the non-linearities involved. Thus, some practical problems making predictions using this theory remain even after the mathematical model is implemented.

\section{Other Sensory Conflict Accounts}

In our everyday life we are exposed to many potentially provocative sensory conflict situations (at least as they are defined by Reason and Brand, 1975). However, we rarely experience any motion sickness (Stoffregen and Riccio, 1991). Thus, Ebenholtz et al. (1994) have argued that " $[\mathrm{w}]$ hat is needed are a priori 
criteria for distinguishing conflict from non-conflict situations" (p. 1034). In recent years, theorists have attempted to precisely specify exactly which types of sensory conflicts cause motion sickness. Below we outline four different hypotheses about what these critical conflicts might be.

\section{The vection conflict hypothesis}

According to this hypothesis, visual illusions of self-motion (vection; see Palmisano et al., 2015) are required to trigger both visually induced motion sickness (VIMS) and cybersickness (Hettinger et al., 1990; Kennedy et al., 1990; McCauley and Sharkey, 1992; Stanney et al., 1998a; Hill and Howarth, 2000; Howarth and Hodder, 2008). When stationary observers are exposed to visual self-motion simulations only some of them become sick. According to Hettinger et al. (1990), what differentiates "sick" from "well" observers is their experience of vection. Even though both groups are exposed to the same sensory conflict (i.e., their visual stimulation indicates self-motion, whereas their inertial stimulation suggests they are stationary), it is only when this multisensory stimulation induces vection that sickness symptoms emerge. This could explain why many sensory conflict situations do not provoke sickness (because they do not induce any, or sufficient, vection). It might also explain why there are individual differences in susceptibility to VIMS and cybersickness since the vection experienced during the same visual motion stimulation can vary quite widely across individuals (Seno et al., 2017).

Empirical evidence. This hypothesis predicts that VIMS and cybersickness should: (1) never occur without vection; and (2) be more likely to occur, and more severe, during stronger vection. Consistent with the hypothesis, the findings of a number of VIMS and cybersickness studies appear to support these predictions (Hettinger et al., 1990; Flanagan et al., 2002; Smart et al., 2002; Bonato et al., 2004, 2005, 2008; Diels et al., 2007; Palmisano et al., 2007; Nooij et al., 2017, 2018; Clifton and Palmisano, 2019; Risi and Palmisano, 2019). However, other studies have reported nonsignificant or negative relationships between vection and sickness (Webb and Griffin, 2002, 2003; Lawson, 2005; Bonato et al., 2008; Ji et al., 2009; Chen et al., 2011; Golding et al., 2012; Keshavarz et al., 2014, 2015; Riecke and Jordan, 2015; Gavgani et al., 2017b; Palmisano et al., 2017, 2018; Palmisano and Riecke, 2018; Kuiper et al., 2019; Teixeira and Palmisano, 2020). Nooij et al. (2017) recently found that the relationship between vection strength and VIMS was stronger when it was examined within (as opposed across) participants. They proposed that such relationships might not always be detectable at the group level-possibly explaining the mixed findings above. However, while the exact relationship between vection and motion sickness is currently unclear, Ji et al. (2009) have shown that VIMS can occur without any vection. This appears to be clear evidence against the strict vection conflict hypothesis (as vection was not required in their study to induce motion sickness).

\section{The subjective vertical conflict hypothesis}

According to this hypothesis: "all situations which provoke motion sickness are characterized by a condition in which the sensed vertical ... is at variance with the subjective vertical as predicted on the basis of previous experience" (Bles et al., 1998, pp. 481-482-see also Bos and Bles, 1998, 2002; de Graaf et al., 1998; Bles et al., 2000; Bos et al., 2008). Bles et al. (1998) also implemented this hypothesis as a mathematical model that constructs: (1) a sensed vertical (by integrating incoming sensory information from the visual, vestibular, and proprioceptive senses); (2) an expected vertical (based on past experiences); and (3) a subjective vertical (which is based on the difference vector between the sensed and expected verticals). According to their hypothesis, it is the vector difference between (1) and (3) that generates motion sickness. Thus, sensory conflicts that do not affect the subjective vertical should not provoke any motion sickness. Like the classical sensory rearrangement theory (from which it evolved), this hypothesis can also explain why motion sickness decreases with repeated exposure to initially provocative stimuli.

Empirical evidence. This hypothesis also provides several readily testable assertions. Consistent with its predictions, motion sickness appears to be more likely when our head moves away from alignment with gravity (Lackner and DiZio, 2006; Thornton and Bonato, 2013; Chen et al., 2016). Bubka and Bonato (2003) have also reported that VIMS increases with (assumed) subjective vertical conflict. When their physically upright observers were placed inside a large rotating drum $^{2}$, VIMS occurred more rapidly when the drum was tilted away from alignment with gravity (by $5^{\circ}$ and $10^{\circ}$ compared to the $0^{\circ}$ control). While at first glance these findings appear consistent with the subjective vertical conflict hypothesis, it is problematic that motion sickness was induced by their $0^{\circ}$ tilt control. According to the hypothesis, no motion sickness should have been induced in this condition, because: (1) the drum had vertical stripes on its inner wall and was rotating smoothly (not wobbling) about a true Earthvertical axis, and (2) the observer's head was always upright and fixed at the center of the drum's rotation. Several other studies have confirmed that VIMS during pure yaw rotation is not due to inadvertent roll or pitch head-movements (Bonato et al., 2005; Nooij et al., 2017). Thus, based on this evidence, subjective vertical conflict also does not appear to be necessary for motion sickness.

\section{The rest frame conflict hypothesis}

According to this hypothesis, motion sickness is caused by conflicting information about what is (and is not) stationary in our surrounding environment (Prothero et al., 1999; Prothero and Parker, 2003). While there are often multiple scene features that could be stationary, it is proposed that only one of them is chosen to serve as a rest frame. This selected rest frame then acts as an important reference for making spatial judgements. According to the rest frame hypothesis: (1) motion sickness should only occur when sensory conflicts prevent the stable perception of a single rest frame (all other sensory conflicts should not be provocative); and (2) adding an independent visual background to displays should reduce this motion sickness (as

\footnotetext{
${ }^{2}$ Their optokinetic drum apparatus rotated in a wobbling fashion when its axis of rotation was tilted away from gravity. However, its rotation was smooth when its tilt was $0^{\circ}$.
} 
this background would be selected as the rest frame and be perceived to be consistent with the available inertial information). The latter prediction suggests that cybersickness should be reduced in HMD based augmented reality ${ }^{3}$ (compared to HMD VR), because users would always be able to see the real world beyond the superimposed synthetic content.

Empirical evidence. Compared to the other theories/hypotheses discussed above, there has been less empirical investigation of the rest frame hypothesis. Consistent with the hypothesis, Prothero et al. (1999) found that cybersickness was reduced when their laboratory wall was also visible in the HMD (compared to when it was blocked from view using a mask). Duh et al. (2004) also found that providing an independent visual background (a distant grid) reduced VIMS in a driving simulator (compared to a no background control). However, while both findings appear consistent with the rest frame hypothesis, they may simply reflect differences in the vection or subjective verticals experienced with and without stationary backgrounds ${ }^{4}$. Further complicating the interpretation of these findings, it appears that cybersickness can also be reduced by superimposing stationary foreground (as opposed to background) surfaces onto virtual environments (Chang E. et al., 2013; Cao et al., 2018). Thus, we conclude that the available data for the rest frame hypothesis are currently inconclusive.

\section{The poison hypothesis}

This hypothesis is an evolutionary account of why motion sickness exists. It is often used to explain the particular signs and symptoms of motion sickness. It is not designed to predict which stimulus conditions will induce it or how it will develop afterwards. Thus, some theorists do not regard it as a competitor for the above explanations of motion sickness. According to this poison hypothesis, motion sickness only occurs when a sensory conflict suggests that we have ingested poison (Treisman, 1977). When swallowed, we will often purge harmful substances from our bodies by vomiting. It is proposed that in some cases responses related to purging (such as stomach awareness, vertigo and dizziness) are triggered by the activity of our visual, vestibular, and proprioceptive control systems. According to Treisman these senses act as an early warning system for the effects of neurotoxins. However, when they register patterns of motion stimulation similar to those during actual intoxication, this can accidently trigger emesis (a reflexive response involving vomiting, nausea, and retching). This hypothesis has recently been extended by Nalivaiko et al. (2014) who propose that: (1) motion sickness triggers defensive hypothermia that acts to cool the sufferer's body; and (2) sweating and changes in skin conductance should therefore provide useful, objective information about the onset and development of motion sickness (Gavgani et al., 2017a,b, 2018).

${ }^{3} \mathrm{HMD}$ AR differs from HMD VR in that the visual environment is only partially produced by the computer (Azuma, 1997). Typically, the virtual content is superimposed over real views of the user's actual environment.

${ }^{4}$ E.g., adding a stationary visual background should have prevented/impaired vection in both studies (Ohmi et al., 1987). Similarly, adding a large background grid could have altered perceptions of the subjective vertical.
Empirical evidence Like sensory rearrangement theory, Treisman's hypothesis has also been criticized for being difficult to test. Consistent with the hypothesis, research has shown that: (1) bilateral vestibular loss not only prevents motion sickness in humans, but it also impairs the vomiting responses of dogs to certain poisons (Kennedy et al., 1968; Money and Cheung, 1983; Cheung et al., 1991); and (2) motion sickness is often accompanied by significant changes in body temperature and skin conductance levels (see Min et al., 2006; Guo et al., 2012; Kim et al., 2014; Gavgani et al., 2017a,b, 2018). It should be noted that vomiting responses are extremely rare in HMD VR. For example, Kingdon et al. (2001) found that only 15 of their 1,028 university student participants vomited during, or after, HMD VR. Given the rarity of vomiting (and retching) responses in HMD VR, Treisman's hypothesis does not appear to be well-suited for understanding this type of cybersickness. It certainly appears to be limited in terms of predicting the occurrence of cybersickness in HMD VR.

\section{The Eye-Movement Theory of Motion Sickness}

According to this theory, motion sickness is triggered by extraocular eye muscle proprioception (not sensory conflict) (Ebenholtz, 1992; Ebenholtz et al., 1994). It is proposed that excessive eye muscle traction $^{5}$ not only stimulates cells in the vestibular nuclei, but also the vagus nerve, which in turn triggers emesis (the reflexive purging response described above). This theory is particularly focused on nystagmusthe compensatory rhythmic eye-movements made in response to prolonged visual/vestibular motion stimulation. However, according to Ebenholtz (1992), "any condition yielding an error in eye-movement control, along with the ensuing feedback and error-correcting signal, is a potential source of motion sickness" (p. 303). Several different oculomotor reflexes attempt to keep vision single, stable, and clear during real/apparent motion. Consider what happens when a person seated on a spinning chair repeatedly rolls their head between upright and tilted toward one shoulder. This not only generates torsional eyemovements (triggered by the otolith organs), but also horizontal and vertical nystagmus (triggered primarily by the cross-coupling of the semicircular canals). According to Ebenholtz et al., the excessive eye muscle traction generated by these complex and competing oculomotor responses should stimulate the vagus nerve. However, while their theory explains why this particular situation should, and does, cause motion sickness (Guedry and Montague, 1961), it is unclear: (1) exactly how much eye muscle traction is required to trigger sickness; and (2) why some eyemovements are provocative and others are not. Thus, the theory appears to suffer from similar problems to sensory rearrangement theory in terms of predicting the occurrence and severity of cybersickness in HMD VR.

\footnotetext{
${ }^{5}$ The theory is based on observations that extraocular muscle traction can trigger other vagal reflexes, such as the oculocardiac reflex (Apt et al., 1973). Gupta (2005) also proposed that extraocular muscles trigger motion sickness but does not refer to Ebenholtz et al. (1994).
} 


\section{Empirical Evidence}

Consistent with the predictions of this theory, VIMS has been found to: (1) increase with the frequency and slow phase velocity of optokinetic nystagmus (Hu and Stern, 1998; Ji et al., 2009); (2) decrease when optokinetic nystagmus is suppressed (Flanagan et al., 2002; Webb and Griffin, 2002; Ji et al., 2009); and (3) be related to the decay rate of this optokinetic nystagmus (Guo et al., 2017). These results do not however provide conclusive evidence for the theory - as the conditions used in these studies would also have altered visual-vestibular conflict and vection (Stern et al., 1990; Hu et al., 1997). Further complicating the story, and contrary to the hypothesis, Nooij et al. (2017) recently failed to find significant relationships between optokinetic nystagmus and VIMS. According to the hypothesis, preventing the observer from making any eye-movements should also prevent them from experiencing motion sickness (since there will be no eye muscle traction signals to trigger symptoms). However, contrary to this key prediction, Money and Wood (1970) found that preventing visual and vestibular eye-movements did not alter the amount of physical motion required to make dogs vomit.

\section{The Postural Instability Theory of Motion Sickness}

While most researchers have assumed that sensory conflict plays an important role in motion sickness, Riccio and Stoffregen (1991) argue that sensory conflict does not actually exist ${ }^{6}$. Instead they propose that prolonged postural instability (of either our body or its segments) is the cause of all types of motion-sickness. According to their postural instability theory, motion sickness occurs when our mechanisms for maintaining postural stability are undermined. It predicts that: (1) individuals who are naturally unstable will be more likely to become sick; (2) this motion sickness will be preceded by increases in postural instability and persist until stability is restored; and (3) motion sickness will be more likely, and become more severe, the longer we remain unstable (Riccio and Stoffregen, 1991; Stoffregen and Smart, 1998; Munafo et al., 2017). While the severity of this sickness is also expected to increase with the degree of postural instability, Riccio and Stoffregen (1991) note that what constitutes postural instability is "not yet well-understood" (p. 213).

\section{Empirical Evidence}

When taken at face value, the evidence for postural instability theory appears to be mixed. While the theory is supported by the findings of many VIMS and cybersickness studies (e.g., Baltzley et al., 1989; Stoffregen and Smart, 1998; Stoffregen et al., 2000, 2008, 2010, 2014; Smart et al., 2002, 2014; Flanagan et al., 2004; Yokota et al., 2005; Bonnet et al., 2006; Merhi et al., 2007; Tanahashi et al., 2007; Reed-Jones et al., 2008; Villard et al., 2008; Chang et al., 2012; Chang C. H. et al., 2013; Koslucher et al., 2015, 2016; Keshavarz et al., 2017; Munafo et al., 2017; Cook et al., 2018; Palmisano et al., 2018; Arcioni et al., 2019; Risi and Palmisano, 2019; Teixeira and Palmisano, 2020), other studies have failed

\footnotetext{
$\overline{{ }^{6} \text { Stoffregen and Riccio (1991) }}$ argue each pattern of multisensory stimulation represents a specific animal-environment situation irrespective of whether the stimulation from the different senses is "redundant" or not.
}

to find relationships between postural instability and motion sickness (Kennedy and Stanney, 1996; Cobb and Nichols, 1998; Warwick-Evans et al., 1998; Cobb, 1999; Akiduki et al., 2003; Dennison and D'Zmura, 2017). In the latter (null finding) studies, postural instability was typically assessed only in terms of the spatial magnitude of the person's movements (e.g., with longer sway paths, larger sway areas and greater positional variability being interpreted as evidence of greater postural instability). However, such measures assume that postural activity is locally self-similar over time. As this is rarely the case, we also need to consider the temporal dynamics of the person's movements when looking for postural precursors of motion sickness (Stoffregen et al., 2010; Koslucher et al., 2016; Munafo et al., 2017). Thus, it is possible that the relationships predicted by this theory could still be found in the data of the latter studies when they are subjected to non-linear analyses (such as detrended fluctuation analysis or recurrence quantification analysis-see Apthorp et al., 2014 and Palmisano et al., 2018 for related discussions).

Research on postural instability theory currently appears to be limited by the state of our knowledge. For example, Keshavarz et al. (2014) recently stated that "it would appear that [Stoffregen and his colleagues] view postural instability theory as consistent with an increase in postural sway prior to [motion sickness], a decrease in postural sway prior to [motion sickness], or an increase in the variability of postural sway prior to [motion sickness]" (p. 660). This suggests that better (or more reliable) methods of identifying postural instability, and increases in postural instability, may be required in the future.

\section{Problems With Existing Theoretical Approaches to Cybersickness}

In the review above, we identified problems with existing theories of cybersickness in terms of their proposed mechanisms, their ability to be tested, or their level of support from the empirical data.

We first considered the sensory conflict theories of motion sickness. Based on our review, we concluded that: (1) it remains difficult to determine a priori which types of sensory conflict will provoke VIMS and cybersickness; (2) classical sensory rearrangement theory and the poison hypothesis lack predictive power and are difficult to test; (3) the data for the rest frame hypothesis are inconclusive; and (4) VIMS and cybersickness can occur without either vection conflict (Ji et al., 2009) or subjective vertical conflict (Bonato et al., 2005). Thus, adopting these sensory conflict approaches has not yet dramatically increased our understanding of the causes of either VIMS or cybersickness.

We also reviewed Ebenholtz's eye-movement theory of motion sickness. In its current form, we believe this also has difficulty precisely predicting the occurrence and severity of cybersickness in HMD VR. While a recent review concludes that the human data for the theory is insufficient (Keshavarz et al., 2014), the animal findings have not thus far been supportive (Money and Wood, 1970).

Finally, we also reviewed the evidence for the postural instability theory of motion sickness. While studies using this approach have been quite successful in identifying which users 
will become sick in HMD VR (Munafo et al., 2017; Arcioni et al., 2019; Risi and Palmisano, 2019; Teixeira and Palmisano, 2020), researchers have not always found its predicted relationships between postural activity and cybersickness (e.g., Dennison and D'Zmura, 2017). As what constitutes postural instability is not yet well-understood, it may be some time before major progress can be made in understanding cybersickness using this approach.

\section{PART 2: A NEW APPROACH FOR STUDYING CYBERSICKNESS IN HMD VR}

Existing sensory conflict, eye-movement and postural instability theories all appear to have difficulties predicting when, and how much, cybersickness will be induced in HMD VR. This may be (at least in part) because they are general theories of motion sickness. That is, they were not specifically created to explain this type of cybersickness. Hill and Howarth (2000) caution that while some cybersickness symptoms can mimic those of other types of motion sickness (e.g., VIMS), their origins are not necessarily the same (see also Stanney et al., 1998b; Lawson, 2014; Palmisano et al., 2017). This was the impetus for us to develop a new approach to understanding and studying cybersickness in HMD VR. As the hypothesis we will outline for this cybersickness is focused on display lag (also known as motion-to-photon latency or end-to-end latency), we will first review the past findings of HMD studies on display lag effects below.

\section{Display Lag Effects on Cybersickness in HMD VR}

Display lag refers to the time required for the user's tracked head movements to change the visual scene presented in their HMD. In HMD VR, this lag is the combined result of sensing, processing, data smoothing, transmission, rendering and frame rate delays (Allison et al., 2001; Wu et al., 2016; Stauffert et al., 2018). Research has shown that: (1) users are sensitive to small changes in display lag (i.e., <20 ms; Ellis et al., 2004; Mania et al., 2004); and (2) display lag can have detrimental effects on user perceptions, performance and well-being (Frank et al., 1988; DiZio and Lackner, 1997; Allison et al., 2001; Meehan et al., 2003). Thus, in recent years, considerable efforts have been made to reduce the effective display lag in modern HMD systems. Nevertheless, some lag remains despite improvements in the technology as well as the use of asynchronous time warping (ATW) and predictive tracking software techniques ${ }^{7}$.

Importantly, display lag is thought to be the main cause of cybersickness in active HMD VR (Howarth and Finch, 1999; Golding, 2016; Kinsella et al., 2016). To test this proposal, researchers have typically injected additional display lag on

\footnotetext{
${ }^{7}$ In ATW, scene views are rendered based on the user's initial head pose, but then shifted (to correct for head motion during rendering) before being sent to display (Van Waveren, 2016). However, as this warping process only corrects for head rotations, stereo and motion cues to 3D layout will be distorted during head translations. Predictive tracking can also reduce the effects of display lag (using dead reckoning, Kalman or alpha-beta-gamma filters to predict where the user's head will be in the future; Kiruluta et al., 1997). However, this can cause the opposite problem (where the user's virtual head can lead its physical position and orientation).
}

top of their system's baseline $\operatorname{lag}^{8}$ and examined its effects on cybersickness. Most of these studies have examined the effects of adding simple constant display lags. However, display lag in HMD VR is not constant, but rather changes over time ( $\mathrm{Wu}$ et al., 2016; Stauffert et al., 2018). Thus, a few studies have also examined the effects of time-varying display lag. Below we review the effects that adding constant, periodic, and jittering display lags have on cybersickness during active HMD VR.

\section{Effects of Adding Constant Display Lag on Cybersickness}

Research has shown that imposing an additional constant lag into the system increases the likelihood and severity of cybersickness (DiZio and Lackner, 1997; Jennings et al., 2000, 2004; Caserman et al., 2019; Feng et al., 2019; Palmisano et al., 2019; Kim et al., 2020). While a small number of studies have failed to find such effects (Draper et al., 2001; Moss and Muth, 2011; Moss et al., 2011), we note that the baseline lags of their systems were already quite high $(\sim 40-70 \mathrm{~ms})$. Our own research has consistently found that cybersickness is increased by imposing additional constant lag into the system (Feng et al., 2019; Palmisano et al., 2019; Kim et al., 2020). Participants in these studies were simulated to either be seated inside a virtual room (Figure 1, Right) or moving forwards through a 3D cloud of randomly positioned objects (Figure 1, Left). They were instructed to make continuous yaw (Feng et al., 2019; Palmisano et al., 2019) or pitch (Kim et al., 2020) head rotations during each 12 second VR exposure. Irrespective of the simulation/environment, or the axis/speed of head rotation, we consistently found that cybersickness severity increased in a monotonic fashion with increases in this constant display lag (Figures 2A-C).

\section{Effects of Periodic Display Lag on Cybersickness}

Periodic variations in display lag can occur during HMD VR due to system clocks, asynchronous processes, buffer times, and sensor drift errors (Wu et al., 2016). Kinsella et al. (2016) and St. Pierre et al. (2015) both examined the effects of periodic variations in display lag on cybersickness. In these studies, participants made natural head movements while completing an object location task. As they moved their heads, the video images of their surroundings were delayed by a variable or constant amount of time before presentation on the HMD ${ }^{9}$. St. Pierre et al. (2015) found that cybersickness was greater when a variable display lag with a frequency of $0.2 \mathrm{~Hz}$ and an amplitude of $100 \mathrm{~ms}$ was added to their baseline system lag of $\sim 70 \mathrm{~ms}$. This $0.2 \mathrm{~Hz}$ display lag was found to be even more provocative for cybersickness when its amplitude varied (from 20 to $100 \mathrm{~ms}$ ) instead of being fixed (at $100 \mathrm{~ms}$ ). Kinsella et al.

\footnotetext{
${ }^{8}$ This refers to the estimated latency of the HMD VR simulation without any added artificial display lag. It is typically estimated by tracking the optical motions of reference and delayed landmarks on the HMD via high-speed digital cameras (Kim et al., 2015; Zhao et al., 2017; Feng et al., 2019). Note: in systems using prediction algorithms and ATW this will estimate the effective lag (not the actual motion-to-photon latency).

${ }^{9}$ Note: this technique only simulates the display lag effects produced by head tracking errors in HMD VR (as HMD tracking data is not actually used to update the display).
} 

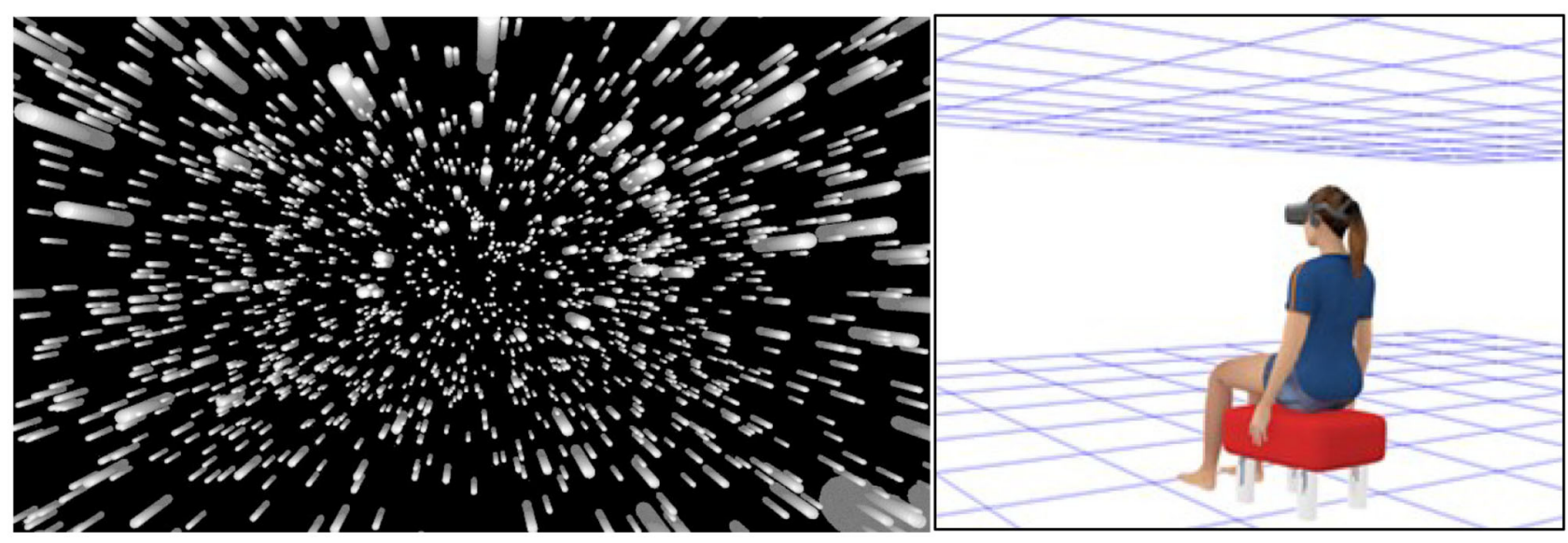

FIGURE 1 | Representations of the simulated virtual environments used in our display lag studies. (Left) In Feng et al. (2019) participants were presented with a radially expanding pattern of optic flow simulating forwards self-motion through a 3D cloud of randomly positioned objects. (Right) In Palmisano et al. (2019) and Kim et al. (2020), the participant was instead simulated to be seated inside a "Tron-like" virtual room (with a wireframe ceiling and ground plane). Note: in the actual displays environmental objects were always blue and the background of the virtual environment was always black (never white).
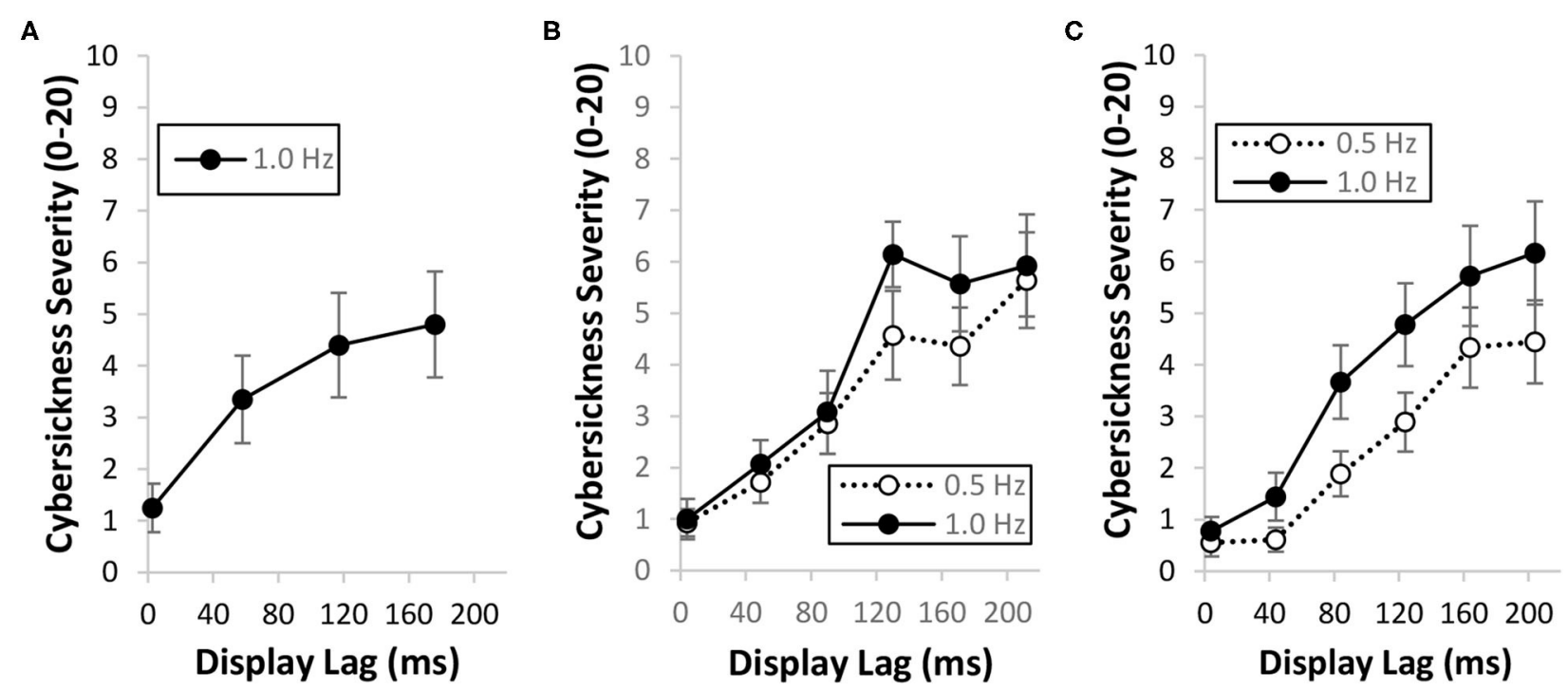

FIGURE 2 | Effects of increasing the mean display lag from $\sim 4$ to 204 ms on cybersickness severity ratings when participants made continuous: (A) yaw head rotations during visually simulated forwards self-motion (Feng et al., 2019); (B) yaw head rotations while they were simulated to be seated inside the "Tron-like" virtual room (Palmisano et al., 2019; binocular viewing condition data only) and (C) pitch head rotations while they were again simulated to be seated inside the "Tron-like" virtual room (Kim et al., 2020). Error bars in each of the three different plots represent standard errors of the mean. Mean data obtained during slow (0.5 Hz) and fast $(1.0 \mathrm{~Hz})$ head movements are also identified.

(2016) subsequently found that the cybersickness induced by this $0.2 \mathrm{~Hz}$ variable lag was more severe than that induced by a $1.0 \mathrm{~Hz}$ variable $\operatorname{lag}^{10}$ - suggesting that both real and apparent motions around $0.2 \mathrm{~Hz}$ might be particularly provocative for motion sickness (Golding et al., 2001).

\footnotetext{
10 That is, cybersickness was more provocative when it took $5 \mathrm{~s}$ (compared to $10 \mathrm{~s}$ ) for the periodic display lag to complete a full cycle. Note: in our studies on the effects of constant display lag, each HMD VR exposure only lasted $12 \mathrm{~s}$.
}

\section{Effects of Jittering Display Lag on Cybersickness}

Recently, Stauffert et al. (2018) also examined the effects of brief latency spikes on cybersickness. Participants in their study performed a virtual search task requiring them to make tracked head movements. They were split into two groups. One group had latency spikes injected into their HMD VR (on top of the baseline system lag of $\sim 36 \mathrm{~ms}$ ), whereas the other group did not. These latency spikes were scheduled to occur randomly (similar to the jittering display lag produced by underperforming 
systems). When a latency spike was scheduled to occur, head tracking data were delayed by a minimum of $1.8 \mathrm{~ms}$ up to a maximum of $60.7 \mathrm{~ms}$ (determined by a probability distribution). Stauffert et al. found that cybersickness was significantly greater for the group with the added latency spikes compared to the control. Thus, it appears that randomly occurring spikes in display lag can also exacerbate cybersickness.

\section{A Hypothesis Specifically Developed for Cybersickness in HMD VR}

As noted above, this hypothesis was created to explain and predict the effects of display lag on cybersickness during active HMD VR. In this situation, display lag will cause inconsistencies between the user's available visual, vestibular, and non-vestibular proprioceptive information about head position and orientation. Consider the person in Figure $\mathbf{3 A}$, who is actively rotating her head in pitch while looking at the real world. When she subsequently makes the same head movement while wearing an HMD, the orientation of her virtual head (Figure 3B, pink) will trail its true orientation (Figure $\mathbf{3 B}$, green) due to the display lag. These Differences in her Virtual and Physical head pose $(D V P)$ could be interpreted as either intersensory conflict or non-redundant multisensory information. However, irrespective of their interpretation, we propose that provocative patterns of $D V P$ are the primary trigger for cybersickness in HMD VR. As can be seen in Figure 3B, the user's DVP will vary over time. Not only will it increase when she initiates a head movement, and decrease sometime after this movement has completed, but it will also vary throughout the movement (due to changes in her head velocity, as well as variations in the display lag, over time). Large changes in this DVP over time will not only make her virtual world appear to swim and oscillate around her (Allison et al., 2001; see Figure 3C for an explanation), but it will also increase the likelihood of cybersickness (Kim et al., 2020). It is proposed that time-varying DVP should still be capable of triggering cybersickness even when it fails to reach the threshold for conscious perception (e.g., when it is generated by brief latency spikes-Stauffert et al., 2018). However, when it does reach consciousness, learned associations between perceived scene instability and past experiences of cybersickness could also act to exacerbate symptom severity.

While the above example is focused on user head rotation in pitch, our research suggests that head rotations in yaw (and presumably also in roll) can generate provocative DVP during HMD VR (Feng et al., 2019; Palmisano et al., 2019). During each of these head rotations, DVP will increase in magnitude and become more variable when: (1) additional (constant or time-varying) display lag is injected into the system; and (2) the user's head-movement speed increases. We therefore expect that both situations should increase the likelihood and severity of cybersickness. We would however expect HMD users to be more tolerant to the same display lags and head speeds during head translations compared to head rotations, because: (1) evidence suggests that vestibular sensitivity is lower for head translations ${ }^{11}$

\footnotetext{
${ }^{11}$ E.g., the otolith-ocular reflex $(\sim 32 \mathrm{~ms})$ tends to be triggered later than the vestibulo-ocular reflex $(\sim 8.6 \mathrm{~ms})$.
}

(Bronstein and Gresty, 1988; Collewijn and Smeets, 2000); and (2) changes in head pose may be more difficult to detect from the complex patterns of visual motion produced by head translation $^{12}$. Thus, head translations are expected to be less likely to produce provocative DVP compared to head rotations. We also expect that once cybersickness is triggered, it will persist until DVP decreases in both magnitude and variability (e.g., after the HMD user minimizes her head motion and keeps it still for some time).

\section{Our Explanation of Cybersickness During Passive HMD VR}

While the DVP hypothesis outlined above is focused on active HMD VR, it can be extended to explain the VIMS and cybersickness experienced during passive viewing conditions. For example, when the HMD user passively views a first-person simulation of a virtual roller coaster ride. Let us first assume that she is physically restrained to prevent any head or body motion. In this case, the roller coaster simulation will still generate large magnitude, time-varying $D V P$ due to the absence of non-visual stimulation confirming her visually simulated selfmotion. This DVP should still increase the likelihood of VIMS even though display lag and head motion does not contribute significantly to it in this case. Now let us assume that the HMD user has been released from her postural restraints and is shown the same simulation again. On her second passive viewing of this virtual roller coaster ride, she will now tend to make small, inadvertent compensatory head-movements (despite her best efforts to keep her head still). If she is asked to stand freely (rather than being seated), she will also tend to sway in response to the roller coaster's visual motion. Under these more ecological passive viewing conditions, additional $D V P$ will be generated by the display lag, which should further increase the likelihood and severity of motion sickness. However, in these head-free and free-standing conditions, any sickness experienced would now be referred to as cybersickness, rather than VIMS (since it would not be due solely to the visual motion; DVP due to head motion and display lag would also contribute to this experience).

\section{Why Is Cybersickness More Severe in HMDs?}

Our DVP hypothesis also explains why this might be the case. Let us compare the visual consequences of the same observer making a tracked head rotation in HMD VR and non-HMD VR. When she makes this head rotation in non-HMD VR (e.g., while viewing a simulation on a large external display), the visual motion expected to accompany her head-movement will be produced immediately and correctly (because it is all generated by her physical head rotation relative to the earthfixed display). That is, she will effectively experience no DVP. By contrast, when she later makes the same head rotation in HMD VR, the expected visual motion will now be delayed by

\footnotetext{
${ }^{12}$ During head rotations, all visual scene elements will move across the user's retinas at similar speeds (irrespective of simulated depth or eccentricity). By contrast, during head translations, there will be a gradient of retinal velocity, with the simulated nearer scene elements moving faster and further than those simulated to be further away (Gibson, 1950).
} 
A

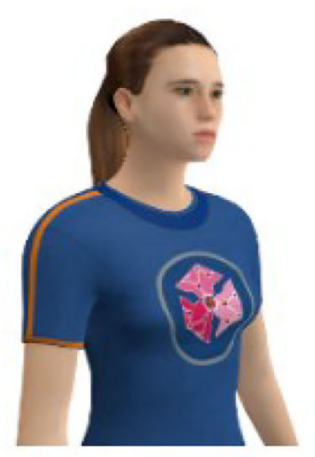

B

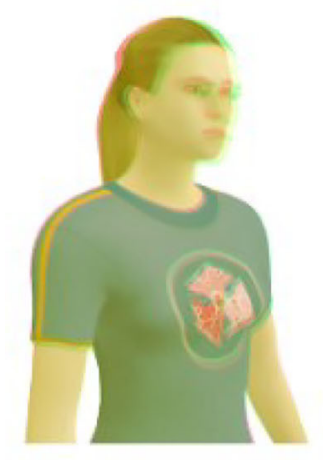

C

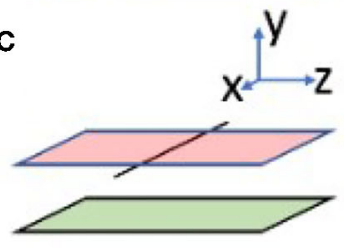

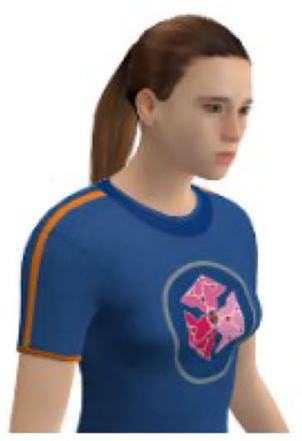
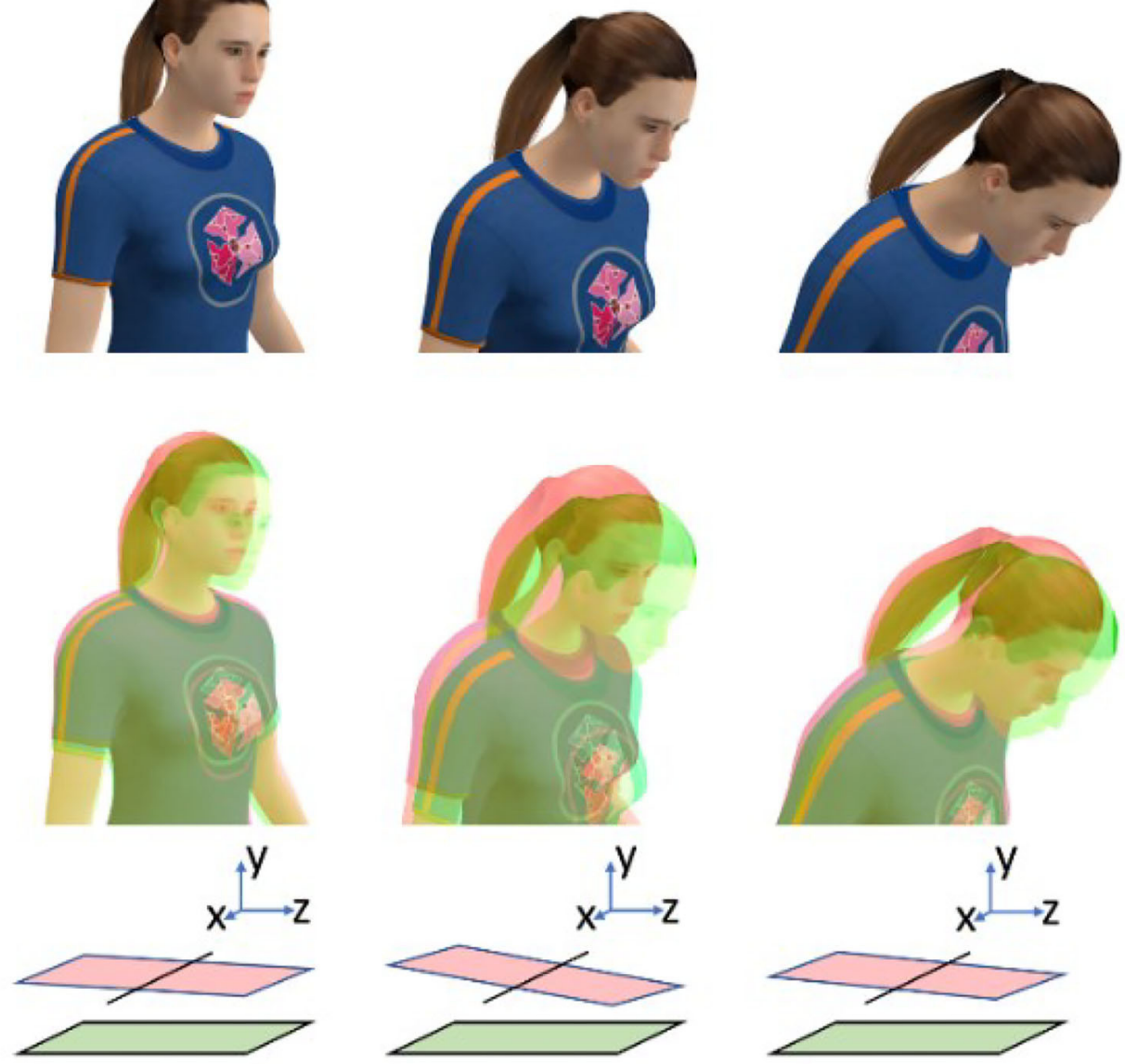
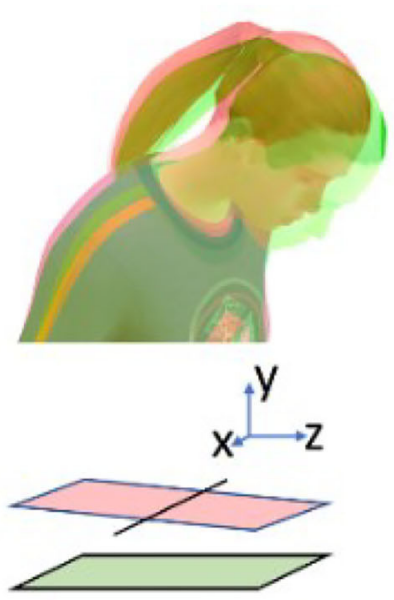

FIGURE 3 | Explanations of the adverse effects of display lag during HMD VR. (A) When this person physically rotates her head in pitch this movement will generate consistent/redundant visual and non-visual information. (B) However, when she makes the same head movements in HMD VR, display lag will generate Differences in her Virtual (pink) and Physical (green) head orientation (DVP). We propose that large magnitude, time-varying DVP will automatically trigger cybersickness in susceptible users. (C) This DVP will also bias the perceived orientation of the ground plane (pink) relative to its true orientation (green). As the DVP (and orientation bias) will change over time, they will be consciously perceived by the HMD user as scene instability.

the system's display lag (since the HMD's screens move with her head and the expected visual motion is computer generated in this case). The longer and more variable this display lag is, the more provocative the DVP should be for cybersickness. However, since there is some (as opposed to no) DVP, this explains why HMD VR is more provocative than non-HMD VR. It is important to note however that such differences in display lag/DVP only occur during head rotations. During head translations, the visual consequences of the user's head motions are similarly delayed for both types of VR (because the expected visual motion parallax ${ }^{12}$ must be computer generated). While this display lag should cause DVP during both types of VR, it should be less provocative than that generated by head rotations in HMD VR (as explained above). Thus, we propose that HMD $\mathrm{VR}$ is more provocative for cybersickness than non-HMD VR primarily because it produces some (as opposed to no) DVP during head rotations.

\section{Summary of Predictions}

Our DVP hypothesis predicts that faster head movements and HMD VR systems with longer/more variable display lags should both increase the likelihood and severity of cybersickness in susceptible users. Active HMD users should therefore be less tolerant to the same display lag when making faster headmovements. These users should also be more likely to become sick when making head rotations as opposed to head translations. During both active and passive HMD VR, we also expect the likelihood of cybersickness to increase when the users' heads are free (as opposed to restrained), and when they are standing freely (as opposed to seated).

All the above predictions are for display lag effects on cybersickness. However, our hypothesis predicts that provocative DVP will sometimes occur when there is minimal display lag (e.g., $4 \mathrm{~ms}$; which is possible using an ideal system with display optimizations, impoverished scene content, as well as ATW 
and predictive tracking software techniques-Feng et al., 2019). As noted above, vection in physically restrained HMD users could produce provocative $D V P$ without any display lag effects. However, movement calibration errors during active HMD VR could also generate provocative time-varying patterns of $D V P$ (e.g., when the user's real-to-virtual head movement gain is not at unity).

\section{Our Approach for Testing the DVP Hypothesis}

Our experiments on cybersickness have often examined the effects of imposing additional constant lags (from 0 to $\sim 200 \mathrm{~ms}$ ) on top of the baseline system lag (of $\sim 4 \mathrm{~ms}$ in Feng et al., 2019, Kim et al., 2020, and Palmisano et al., 2019). The different display lag conditions used in these studies were created using the memory buffer method described in the next section Memory Buffer Method for Imposing Additional Constant Display Lag. Our use of this method also allowed us to objectively estimate the user's DVP throughout each trial (based on comparisons of their tracked head pose at different times in the trial). The exact procedure we used to calculate this DVP time series data is described in the following section Method for Estimating DVP Due to Display Lag.

\section{Memory buffer method for imposing additional constant display lag}

Before each trial, a circular memory array (of element length N) was constructed to store the user's head tracking data (see Figure 4). The user's head position and orientation data were then continuously sampled from the HMD sensors over the course of the trial. These data were written to the memory array on every single frame.

In the example shown in Figure 4 below, current head position and orientation data are being written to the array element located at index $\mathbf{t}_{\mathbf{i}}$. They will be held there until all head pose data written earlier have been used for rendering. Next, the array counter will be incremented to read the head pose data stored at index $\mathbf{t}_{\mathbf{i}+1}$. These data from $\mathbf{t}_{\mathbf{i}+1}$ are then used to update the user's virtual environment.

As can be seen in Figure 4, small constant increments in display lag can be added to the system simply by increasing the number of elements in this circular memory array. In the case of a single element array $(N=1)$, there will be no additional imposed display lag (i.e., the scene updates should only be delayed by the system's baseline lag). However, when using an 18-element array there will be an additional delay of 18 frames on top of the system's baseline lag (resulting in $\sim 200 \mathrm{~ms}$ imposed lag $+\sim 4 \mathrm{~ms}$ baseline lag $=\sim 204 \mathrm{~ms}$ in our experiments; as HMDs with a $90 \mathrm{~Hz}$ refresh rate were used; either the Oculus Rift CV1 or the Oculus Rift S).

\section{Method for estimating DVP due to display lag}

Let us assume that a participant made continuous oscillatory pitch head movements at $0.5 \mathrm{~Hz}$ during a VR exposure lasting $12 \mathrm{~s}$ (similar to one of the conditions in our recent Kim et al., 2020 study). After the participant completed the trial, we would first use the rotation vectors from their HMD sensor data to build a 3D view matrix for each eye (to account for their interocular separation-see Equation 1). Then we would obtain their yaw, pitch, and roll angular head orientation data from this view matrix (in Euler angles) using the mathematical transformations shown in Equations (2)-(4):

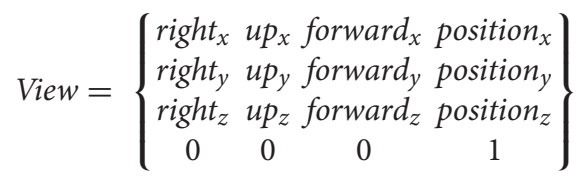

$$
\begin{aligned}
& \text { Yaw }(\theta)=\operatorname{atan}\left(-\frac{\text { right }_{y}}{\text { right }_{x}}\right) \\
& \text { Pitch }(\varphi)=\operatorname{atan}\left(\frac{u p_{z}}{\text { forward }_{z}}\right) \\
& \operatorname{Roll}(\psi)=\operatorname{atan}\left(\frac{\text { right }_{z}}{\operatorname{sqrt}\left(u p_{z}^{2}+\text { forward }_{z}^{2}\right)}\right)
\end{aligned}
$$

As the participant was asked to make pitch head movements in this case, we could estimate their DVP using only the pitch orientation data for the trial (ignoring the smaller differences in yaw and roll head orientation shown in Figure 5A $)^{13}$. However, we would first need to know the display lag for the trial. As added lag was injected into the system using the memory buffer method outlined in the previous section, this could be approximated as the temporal offset between the time of writing to, and the time of reading from, the memory buffer. In other words, the added lag would be the element length $\mathbf{N}$ of the memory array used for that trial. This temporal offset (in frames) would then be used to simulate the user's virtual head orientation in pitch throughout the trial (Figure 5B). At each instant, the participant's physical head orientation would be estimated as their recorded pitch head orientation for that time, and his/her virtual head orientation would be estimated as their recorded pitch head orientation from a time $\mathrm{N}$ frames earlier. The DVP experienced at this time could then be calculated as the difference in head orientation between these two estimates. Figure 5C shows an example of the DVP time series data estimated from the original data shown in Figure 5A. Similarly, Figure 5D shows the unsigned magnitudes of this DVP. Based on our hypothesis, we would expect cybersickness to be more likely and severe as the peak and standard deviation of this estimated $D V P$ increases.

\section{Empirical Support for the DVP Hypothesis}

Kim et al. (2020) recently used the approach outlined above to test our DVP hypothesis for cybersickness. In this study, 30 participants were asked to make continuous oscillatory pitch head movements (Figure 6, Top Right) while viewing a "Tronlike" virtual room environment through an Oculus Rift CV1 HMD (Figure 6, Left). On different trials: (1) we examined the effects of imposing additional constant lags (ranging from 0 to $\sim 200 \mathrm{~ms}$ ) on top of the baseline system lag (of $\sim 4 \mathrm{~ms}$; using the memory buffer method described in the section Memory Buffer Method for Imposing Additional Constant Display Lag);

${ }^{13}$ DVP could also be estimated based on orientation differences across all three axes, or even based on the differences in position and orientation across these axes. 

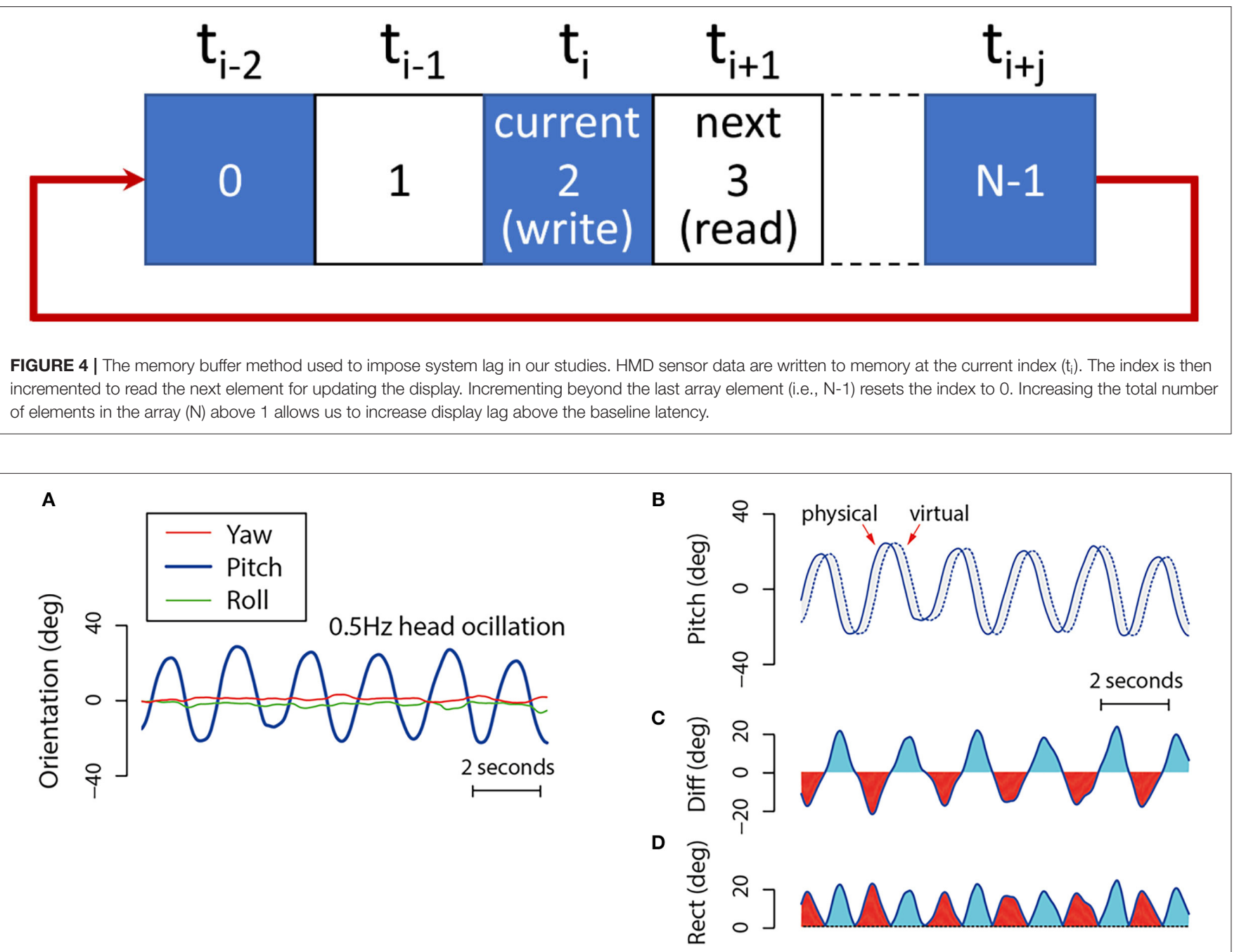

FIGURE 5 | This depicts the method used to estimate DVP in Kim et al. (2020). (A) An example of the yaw, pitch and roll head orientation time series data produced by a participant oscillating their head in pitch for $12 \mathrm{~s}$. (B) Shows both the recorded (physical) and estimated virtual (virtual) pitch head orientation data for the participant across the trial. In order to estimate the effects of display lag on virtual head pose, these simulated data were assumed to be the same as the recorded head pose from a time $\mathbf{N}$ frames earlier. (C) Shows the per-sample DVP over the course of the entire trial. (D) Shows the unsigned differences in this DVP over the same time period.

and (2) our participants made either fast $(1.0 \mathrm{~Hz})$ or slow $(0.5 \mathrm{~Hz})$ head movements with approximately equal amplitudes. Head pose time series data (obtained from the HMD's sensors) and cybersickness severity ratings (using the Fast Motion Sickness scale; Keshavarz and Hecht, 2011 $\mathrm{b}^{14}$ ) were recorded for each trial. After participants completed the experiment, we then estimated their DVP time-series data for each trial using the method outlined in the section Method for Estimating DVP Due to Display Lag. As can be seen in Figure 7A, the unsigned mean of this DVP increased with both the imposed display lag and the participants' head speed for the trial. Consistent with our DVP hypothesis, we reported a strong positive linear relationship between mean unsigned DVP and cybersickness severity (Figure 7B). Since

\footnotetext{
${ }^{14}$ Cybersickness was also confirmed using the Simulator Sickness Questionnaire (Kennedy et al., 1993).
}

mean unsigned DVP also increased with the participant's head speed, the finding that cybersickness was greater in the $1.0 \mathrm{~Hz}$ (compared to the $0.5 \mathrm{~Hz}$ ) conditions was also interpreted as support for our hypothesis.

In this paper we propose that large magnitude, time-varying DVP is the trigger for cybersickness. However, Kim et al. (2020) only reported mean unsigned DVP in their recent cybersickness study. Thus, we re-examined their data to see whether peak DVP (Figure 7C) and the standard deviation of the DVP (Figure 7D) also predicted cybersickness [For a description of these new analyses and statistics please see our Supplementary Materials document: "1. Relationships between DVP and Cybersickness in the Kim et al. (2020) study"]. Consistent with our hypothesis, both the peak and the standard deviation of the DVP were found to have significant positive linear relationships with cybersickness severity. 


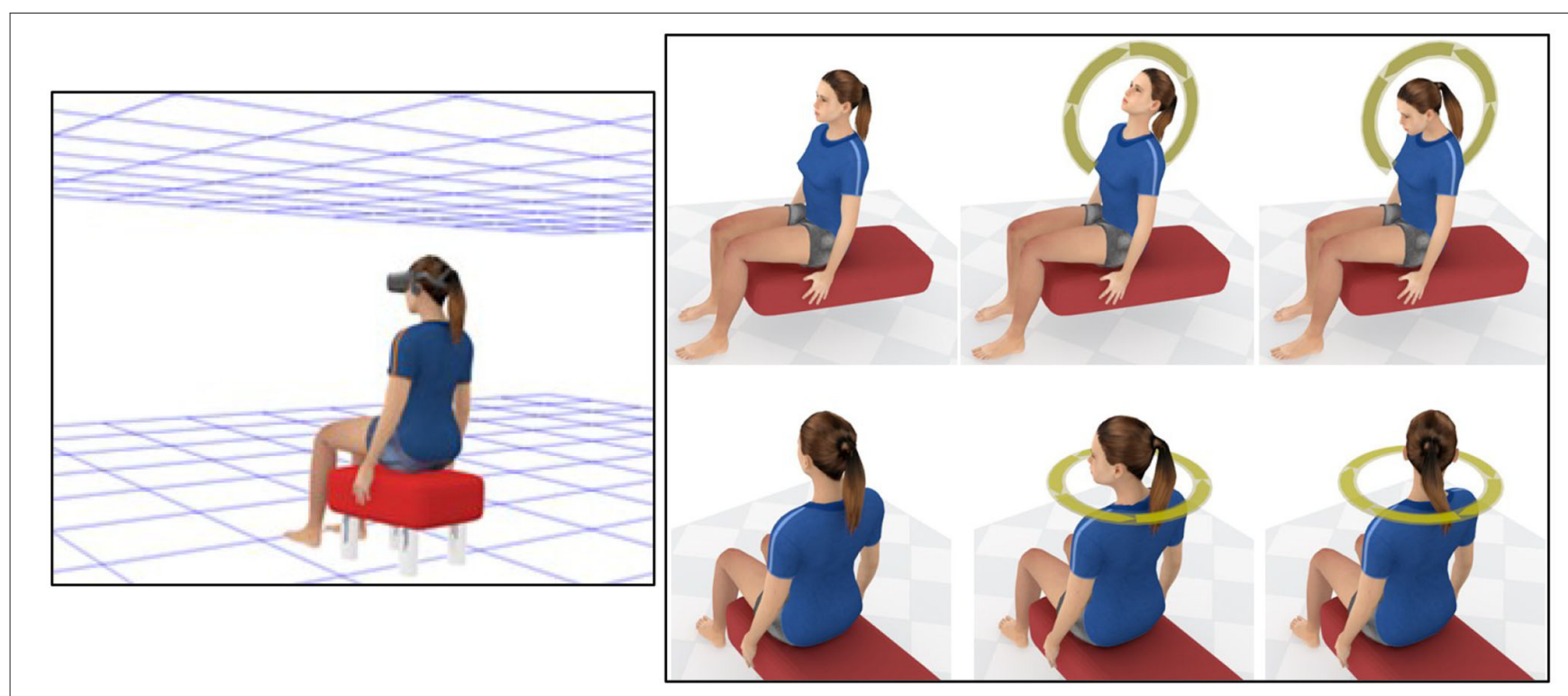

FIGURE 6 | (Left) Representation of the "Tron-like" virtual environment used in both Kim et al. (2020) and Palmisano et al. (2019). Note that the background used in the actual simulation was black (not white). (Top Right) This shows the continuous pitch head-movements made during HMD VR in the Kim et al. (2020) study; (Bottom Right) This shows the continuous yaw head movements made in the Palmisano et al. (2019) study.

The above findings (and our new analysis) provide evidence that $D V P$ can be used to predict cybersickness in HMD VR during pitch head rotations. To investigate whether $D V P$ can also predict cybersickness during yaw head rotations, we re-examined the data from another of our recent studies. In this Palmisano et al. (2019) study, 14 participants made continuous oscillatory yaw head rotations (Figure 6, Bottom Right) while viewing the same "Tron-like" virtual room through an Oculus Rift CV1 HMD (Figure 6, Left). The binocular viewing conditions of this experiment were otherwise identical to those in the Kim et al. (2020) study. After estimating the DVP time series data for each trial, we calculated the unsigned mean, peak and standard deviation of this $D V P$, and used detrended fluctuation analysis (DFA) to also examine its temporal dynamics. The DFA scaling component $(\alpha)$ was calculated for each trial (this indicates the relative distribution of the variance in the DVP across different timescales ${ }^{15}$ ). We then investigated whether each of these four different $D V P$ indices were able to predict cybersickness severity [For a description of these analyses and statistics please again see our Supplementary Materials document: "2. Relationships between DVP and Cybersickness in the Palmisano et al. (2019) study"]. Consistent with Kim et al. (2020), we again found significant positive linear relationships between the mean unsigned DVP and cybersickness severity (Figure 8A). We also found significant positive linear relationships between peak DVP and cybersickness severity (Figure 8B) and between

\footnotetext{
${ }^{15}$ DFA $\alpha$ values greater than 0.5 indicate that an autocorrelation has occurred at some timescale in the data. An $\alpha$ of 1 represents the maximum possible selfsimilarity. As $\alpha$ increases above 1, a greater proportion of the fluctuations occur at longer time scales. As $\alpha$ was always 1.47 or greater in Palmisano et al. (2019), the fluctuations in the DVP over time appear to be most similar to Brownian noise.
}

the standard deviation of the DVP and cybersickness severity (Figure 8C). Positive relationships were also observed between the DFA $\alpha$ values and cybersickness severity ratings (Figure $\mathbf{8 D}$ ). However, in contrast to the other three DVP measures, these relationships involving DFA $\alpha$ did not remain significant after statistical corrections were made for multiple comparisons.

\section{Could Our Findings Be Explained by Other Theories of Motion Sickness?}

In the studies reviewed above, longer imposed display lags and faster user head speeds were both shown to increase the magnitude and variability of the HMD user's DVP (Figure 7A). Consistent with the predictions of our DVP hypothesis, both manipulations also resulted in more severe cybersickness. Below we consider whether these cybersickness findings could also be explained by any of the other theories of motion sickness.

\section{Vection conflict hypothesis}

According to this hypothesis: (1) vection is required for cybersickness; and (2) cybersickness severity should increase with vection strength. However, in both the Kim et al. (2020) and Palmisano et al. (2019) studies, participants were always simulated to be seated and stationary inside a virtual room. The only motion stimulation they experienced during their brief $12 \mathrm{~s}$ exposures to HMD VR was generated by their own physical head motions (as well as the visual consequences of the display lag). While they should have experienced little to no vection under these conditions, they still reported cybersickness in both studies. Interestingly, their cybersickness severity ratings were quite similar to those in Feng et al. (2019), even though the conditions in that study were much more likely to induce 


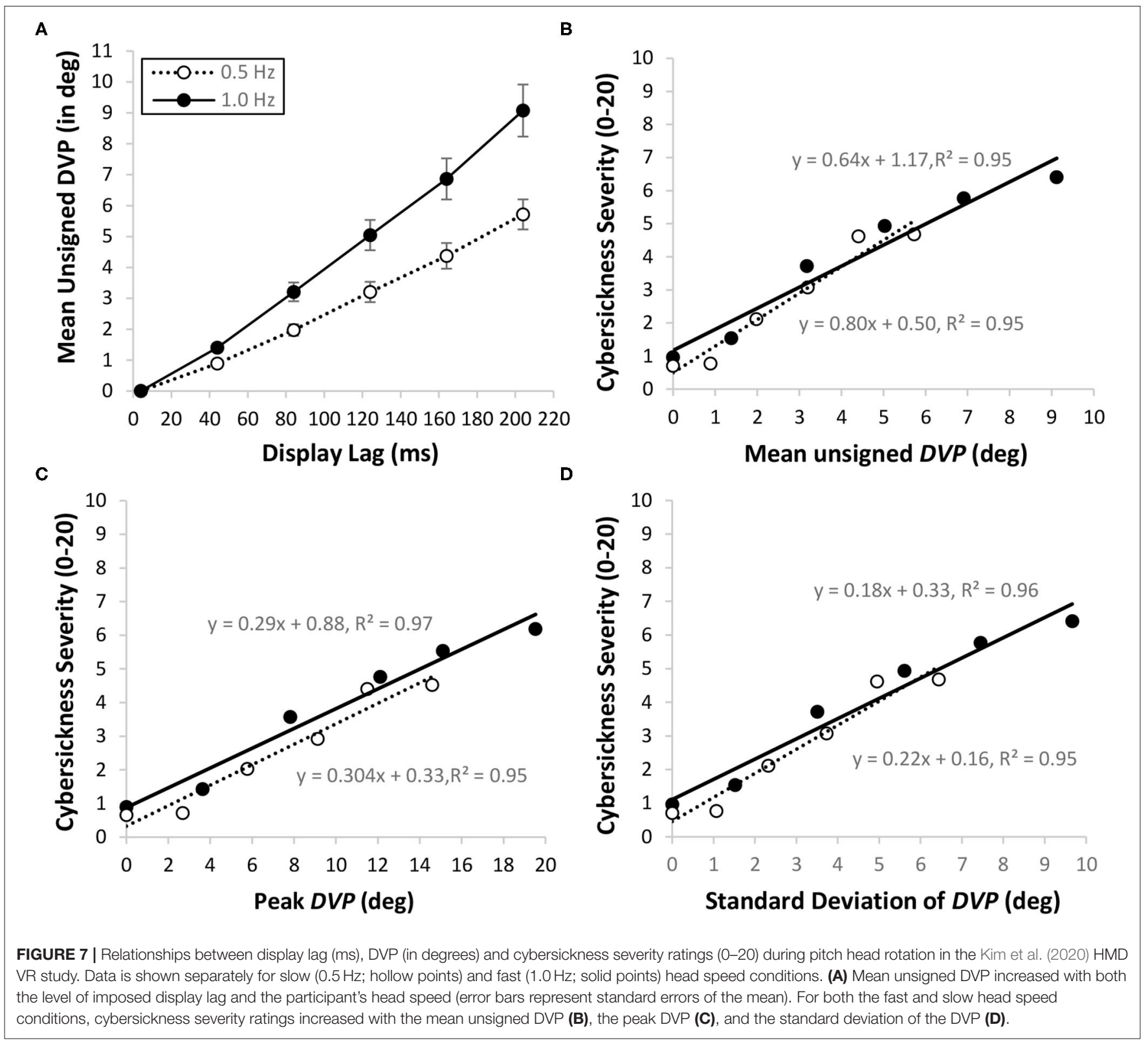

vection $^{16}$ (Figures 2A-C show the cybersickness ratings for the Feng et al., 2019, Palmisano et al., 2019 and Kim et al., 2020 studies, respectively). Thus, the findings of the Kim et al. (2020) and Palmisano et al. (2019) studies do not appear to support either prediction (1) or (2) of this vection conflict hypothesis.

\section{Subjective vertical conflict hypothesis}

According to this hypothesis, only sensory conflicts that affect the subjective vertical should cause cybersickness. In the Kim et al. (2020) study, participants made pitch head movements, whereas in Palmisano et al. (2019) they made yaw head movements.

\footnotetext{
${ }^{16}$ The participants in this study were simulated to be moving forwards at a constant velocity.
}

However, only pitch head movements should have produced significant instability in their perceived orientation (and that of the ground) relative to gravity. Therefore, the subjective vertical conflict hypothesis predicts that: (1) cybersickness should be more severe in the Kim et al. study; and (2) any cybersickness in the Palmisano et al. study would be due to inadvertent pitch and roll (but not yaw) head motions. Contrary to both predictions, pitch rotation conditions were not more provocative than yaw rotation conditions. In fact, cybersickness severity ratings were similar for equivalent levels of display lag and head speed (see Figures 2B,C). As was noted above, significant cybersickness was also found in the Feng et al. (2019) study (see Figure 2A). Like the Palmisano et al. (2019) study, this was also focused on the effects of display lag on cybersickness during yaw head rotations. Thus, the findings of the Kim et al. (2020) and Palmisano et al. (2019) 
A

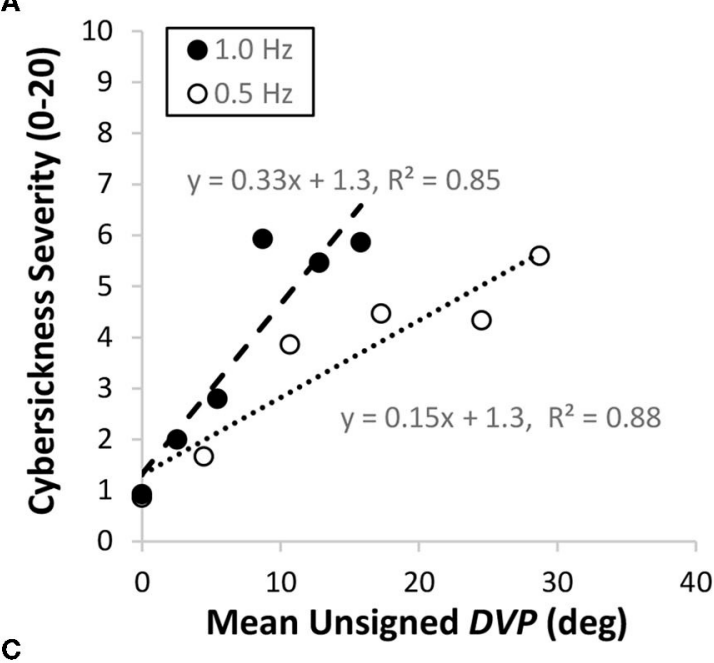

C

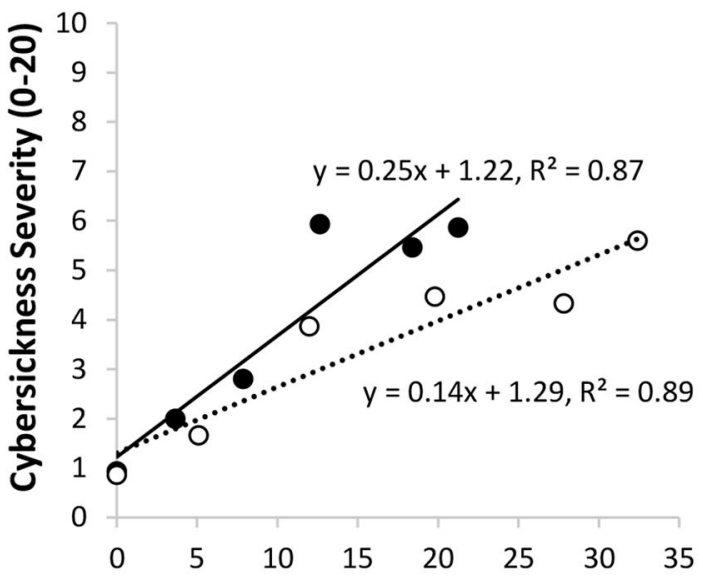

Standard Deviation of DVP (deg)
B
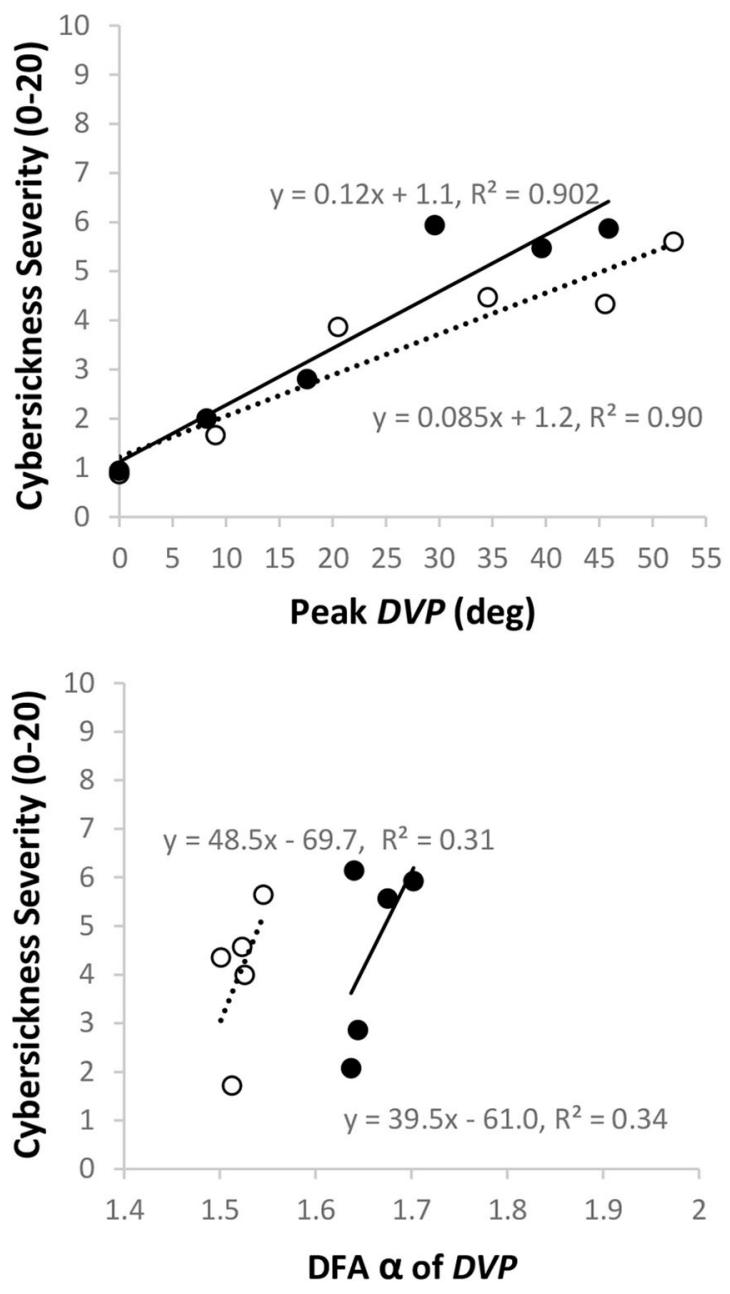

FIGURE 8 | Data from the binocular viewing conditions of the Palmisano et al. (2019) HMD VR study. Participants in this study made continuous slow (0.5 Hz; hollow points) and fast $(1.0 \mathrm{~Hz}$; solid points) head rotations in yaw. For both head speed conditions, cybersickness severity ratings increased with the mean unsigned DVP (A), the peak DVP (B), the standard deviation of the DVP (C), and the DFA $\alpha$ for the DVP (D).

studies do not appear to support either prediction (1) or (2) of the subjective vertical conflict hypothesis.

\section{Rest frame conflict hypothesis}

According to this hypothesis, cybersickness should only occur when sensory conflict prevents the stable perception of a single rest frame. In both the Kim et al. (2020) and Palmisano et al. (2019) studies, participants only saw a wireframe ceiling and ground plane (the rest of their virtual environment was completely black; see Figure 6, Left). These environmental surfaces were always simulated to be stationary. Thus, since all of their visual motion was produced by the user's head motions, there should have been little or no rest frame conflict and cybersickness in either study (as both surfaces should have appeared to move together in a rigid fashion, they could have effectively served as a single rest frame). However, contrary to the predictions of this hypothesis, cybersickness was still found to occur in both studies.

\section{The poison hypothesis}

According to this hypothesis, vomiting, retching, and related responses should occur during sensory conflicts which suggest we have ingested poison. None of the participants vomited in either the Kim et al. (2020) or the Palmisano et al. (2019) studies. Also, as noted previously, the poison hypothesis cannot be used to make testable predictions about the effects of stimulus factors or the development of predicted symptoms.

\section{The eye-movement theory}

This theory predicts that cybersickness is triggered by excessive extraocular eye-muscle traction. As we did not record participant eye-movements in the Kim et al. (2020) and Palmisano et al. (2019) studies, it is not possible to directly relate their DVP and cybersickness findings to this theory. Increasing the participant's 
DVP (by increasing the display lag or having them make faster head movements) should have altered their eye-movements and retinal motion, which could conceivably have increased the likelihood of cybersickness. However, we note that some sickness was still experienced in the slow head movement, baseline display lag conditions of both studies (see Figures 2B,C). It does not seem likely that these conditions would have produced enough eye-muscle traction to trigger such symptoms.

\section{Postural instability theory}

As we did not record postural activity during HMD VR in either study, we cannot directly relate their DVP and cybersickness findings to this theory. However, it is possible that the preconscious pickup of time-varying DVP triggered postural activity and instability in our participants, which in turn generated the cybersickness reported in the Kim et al. (2020) and Palmisano et al. (2019) studies. This possibility will be discussed in more detail in the section DVP and Individual Differences in Cybersickness below.

\section{Reconciling our DVP Hypothesis With Well-Known Findings}

There are still several well-known cybersickness findings that our hypothesis has yet to explain. Below we attempt to reconcile two of these findings with our DVP hypothesis.

\section{DVP and adaptation to cybersickness}

Currently our DVP hypothesis does not have a specific mechanism that explains why cybersickness adapts/habituates with repeated exposures to provocative stimuli. However, if our proposed trigger for cybersickness $(D V P)$ is treated as a sensory conflict involving head pose, then a neural mismatch type explanation (see the section on Sensory Rearrangement Theory) could work for our hypothesis as well. When users move in HMD VR, it would be assumed that their DVP is continuously compared to the expected multisensory stimulation for the movement. Thus, upon first entering HMD VR, users should be more likely to experience cybersickness, because at this time, their expected stimulation will be what they would normally experience in the real world. This should result in a significant neural mismatch, as the actual stimulation they are receiving has DVP due to display lag. However, with repeated exposures to HMD VR, users should gradually become recalibrated to this $D V P$, resulting in a little less neural mismatch and cybersickness on each subsequent exposure. If this explanation is valid, then according to our hypothesis, it should be easier to adapt to the DVP produced by adding constant and periodic display lags to HMD VR than to the DVP produced by random latency spikes. This would therefore be an important topic for future research on our DVP hypothesis.

\section{DVP and individual differences in cybersickness}

When presented with the same HMD VR simulation, some users are much more likely to become sick, and also experience this sickness more severely, than others (Munafo et al., 2017; Arcioni et al., 2019; Cao et al., 2019; Clifton and Palmisano, 2019; Risi and Palmisano, 2019; Curry et al., 2020; Teixeira and Palmisano, 2020). Currently, our DVP hypothesis does not have a specific mechanism to explain individual differences in cybersickness during HMD VR. In principle, such findings could be due to differences in user sensitivities to motion, visualvestibular conflict or even the specific patterns of $D V P$ produced by HMD VR. Vestibular thresholds for angular acceleration appear to vary quite widely in healthy individuals across studies (e.g., from 0.035 to $4 \mathrm{deg} \mathrm{s}^{-2}$; Clark and Stewart, 1970; Guedry, 1974; MacNeilage et al., 2010). This is (in part) because there appear to be significant individual differences in vestibular motion detection/discrimination thresholds (Clark and Stewart, 1970; MacNeilage et al., 2010; Roditi and Crane, 2012; Valko et al., 2012). Thus, one possibility is that users who are more sensitive to physical head pose/motion are also more susceptible to cybersickness due to DVP.

Alternatively, it may be that DVP can only explain withinsubject effects on cybersickness (such as the effects of increasing the magnitude of the display lag or the speed of the user's head movement). In order to explain known/possible age (e.g., Cao et al., 2019), sex (e.g., Munafo et al., 2017) and other betweensubject effects on cybersickness in HMD VR, we may need to look to other existing theories for inspiration. For example, if $D V P$ is treated as non-redundant multisensory stimulation, then our hypothesis is potentially compatible with the postural instability theory of motion sickness. According to this view: (1) the preconscious pickup of large amplitude time-varying DVP could signal that the user's head pose is unstable; and (2) the automatic postural activity produced by this DVP could then increase the likelihood of him/her becoming posturally unstable and cybersick. Individual differences in the user's natural stability could then determine how destabilizing these automatic postural responses are, and how quickly he/she can return to a state of relative stability/wellness. Consistent with this idea, several recent HMD VR studies have found that individuals who are naturally unstable are more likely to become sick (Munafo et al., 2017; Arcioni et al., 2019; Risi and Palmisano, 2019; Teixeira and Palmisano, 2020). Each of these studies first examined their participants' spontaneous postural activity when standing quietly before entering HMD VR. In all four studies, pre-exposure postural activity was found to differ between the participants who later became sick and those who remained well during HMD VR. These findings suggest that it might be possible to predict susceptibility to cybersickness (before any exposure to HMD VR) based on individual differences in natural spontaneous postural stability.

\section{Benefits of Studying Cybersickness Using DVP}

Below, we compare our approach to studying cybersickness to traditional approaches based on sensory conflict and postural instability.

\section{Comparing DVP and conflict approaches}

If one treats $D V P$ as an intersensory conflict regarding head pose, then our proposed approach has some advantages over traditional conflict-based approaches to cybersickness. Instead of merely speculating about the presence, or degree, of sensory conflict in a condition (like many past studies), our approach 
allows researchers to quantify the amount of $D V P$ produced during each exposure to HMD VR. This metric is an objective measure of the stimulation rather than an internal model of the HMD user's sensory processing. In the sections on Memory Buffer Method for Imposing Additional Constant Display Lag and Method for Estimating DVP Due to Display Lag, we show how objective estimates of the DVP produced by display lag can be calculated from the participant's own head tracking data for each trial. Using such estimates, it should be possible to determine whether a particular VR condition is likely to be provocative (or not) for cybersickness. This determination could be based on the patterns of $D V P$ that such conditions: (1) have generated in the past with other HMD users, or (2) are currently being generated while the user is actively experiencing HMD VR.

\section{Comparing DVP and postural instability approaches}

Our approach also appears to have some practical advantages over approaches using postural instability. According to postural instability theory, motion sickness is caused by prolonged postural instability of either the body or its segments. So, researchers using this approach must carefully examine both the spatial magnitudes and the temporal dynamics of their users' head, body and limb movements during HMD VR. There is also another obstacle to understanding cybersickness based on postural instability. Unfortunately, what constitutes postural stability and instability is currently not well-understood (e.g., there are more than nine different proposed operational definitions or "signatures" of postural instability; Riccio and Stoffregen, 1991). This makes it difficult to determine whether a change in the user's postural activity represents an increase in their postural instability or not. For example, an increase in their gross body motion alone would not be sufficient (as the postural activity in this case might be well-controlled/deterministic as opposed to random/chaotic). Researchers would therefore need to look for additional evidence of an increase in postural instability (such as changes in physiological tremor, spreading instability across joints, or increasing variability in the phase relations between the various degrees of freedom involved in the movement).

By contrast, our DVP approach to cybersickness is only focused on the user's head movements, not on the movements of their body or their limbs. This focus on the head seems particularly appropriate for HMD VR, given the greater influence that tracked head movements have on the user's point of view and avatar. We have shown that cybersickness can be predicted by simple summary measures of time-varying $D V P$ (e.g., its mean, peak and standard deviation). If the HMD user is asked to make head rotations about a single axis (e.g., pitch), these predictions appear to hold even when $D V P$ is only calculated using the head orientation data for that same axis (i.e., ignoring any differences in yaw and roll head orientation in the case of this example).

Thus, as our DVP hypothesis provides a simpler operational definition of the provocative stimulation during HMD VR, it should be much easier to identify and examine possible $D V P$-based precursors of cybersickness compared to possible precursors of sickness based on postural instability.

\section{Future Directions and Implications Future studies on DVP and cybersickness}

In this paper, we proposed that cybersickness in HMD VR is triggered by large magnitude, time-varying DVP. However, a considerable amount of research still needs to be done to investigate and validate our DVP hypothesis.

Identifying precursors of cybersickness based on DVP. Our research to date has focused on the relationship between $D V P$ and cybersickness severity. We still need to determine the exact nature of the changes in DVP that initially trigger this cybersickness. In such a study, participants would need to remain in active HMD VR until either their first report of cybersickness or the simulation times out. Then the estimated DVP for the trial could be analyzed using a windowing procedure similar to that used by Dong et al. (2011). For sick participants, we would calculate summary and temporal dynamic measures of the $D V P$ for the first, middle and final Y seconds of the trial. For those who remained well, we would also calculate those measures for the same average time windows. This would allow us to identify triggering changes in the $D V P$ by: (1) comparing the sick participant's $D V P$ measures in their final window to those in their first and middle windows; and (2) comparing DVP measures in the final windows for sick and well participants.

Periodic and jittering display lags. In our studies to date $D V P$ was always manipulated by introducing additional constant display lag into the system. Research is therefore still needed to determine the effects that periodic and jittering display lag have on $D V P$ and cybersickness during active HMD VR. Such studies could use a similar approach to that outlined in the section on Our Approach for Testing the DVP Hypothesis. Researchers could inject artificial periodic/jittering lag on top of the HMD's baseline system lag, and then, using time-stamped information about the added lag, they could estimate the DVP experienced at each instant from the user's own head tracking data. Summary and temporal dynamics measures based on this DVP could then be compared to the user's cybersickness ratings.

Other types of head movements. Thus far, we have only examined the relationship between DVP and cybersickness when users make continuous yaw and pitch head rotations. Thus, we still need to examine the effects of head rotations in roll and head translations during HMD VR. While we expect that the relationships observed for yaw and pitch head movements should also generalize to roll, it is predicted that the DVP produced by head translations will be substantially less provocative for cybersickness.

For angular head movements like those made in our HMD VR studies (Feng et al., 2019; Palmisano et al., 2019; Kim et al., 2020), frequency also appears to be important. For example, Grabherr et al. (2008) found that the vestibular thresholds for discriminating left-right yaw rotations were quite similar within the range of $0.5-5 \mathrm{~Hz}(\sim 0.6-0.7 \mathrm{deg}$ $\mathrm{s}^{-1}$ ). However, vestibular discrimination thresholds were much higher for movements of $0.2 \mathrm{~Hz}$ or less (e.g., the minimum velocity required for direction discrimination was $2.8 \mathrm{deg} \mathrm{s}^{-1}$ 
on average for a $0.05 \mathrm{~Hz}$ movement). The relative precision of vestibular (compared to visual) thresholds also appears to depend on head movement frequency. For example, Karmali et al. (2014) found that for physical rotations between 0.1 and $1 \mathrm{~Hz}$, vestibular thresholds appear to be higher than visual thresholds for discriminating roll motion direction. However, this relationship appears to reverse for physical movements above $2 \mathrm{~Hz}$, with vestibular thresholds appearing to be lower than visual thresholds. So it will be important to examine whether the current cybersickness findings for 0.5 and $1.0 \mathrm{~Hz}$ generalize to other head movement frequencies.

It will also be important to examine the relationship between DVP and cybersickness during free/naturalistic head movements-e.g., using virtual search tasks similar to those in Kinsella et al. (2016), St. Pierre et al. (2015) and Stauffert et al. (2018).

Different ways to estimate DVP. In our studies to date we asked participants to rotate their head in either pitch or yaw, and then we estimated their DVP based simply on the changes in head orientation along that same axis. However, this approach ignored the DVP produced by their linear head motions and any head rotations about the other two axes. Future research and analysis are therefore needed to determine whether calculating the combined DVP across all three axes $(\mathrm{x}, \mathrm{y}, \mathrm{z})$ and both types of head movements (rotation and translation) improves the prediction of cybersickness in HMD VR.

Effects of DVP on eye-movements and postural instability. Finally, the effects of $D V P$ on both the user's eye-movements and their postural stability also need to be investigated. For example, eyemovement recordings made during HMD VR could be used to determine the extent to which DVP generates nystagmus and errors in gaze holding during head rotation. Similarly, center of foot pressure recordings during HMD VR could be used to determine how the user's DVP affects their overall postural activity, as well as their head movements and experiences of cybersickness.

\section{Possible role of DVP in mitigating cybersickness}

In a laboratory context, DVP should allow researchers to precisely predict the effects that different HMD VR conditions will have on cybersickness. Researchers could extrapolate the likelihood/severity of cybersickness in a particular experimental HMD VR condition based on the user's own DVP and sickness data (e.g., obtained during past exposures to similar conditions/simulations). However, we believe that DVP could also be useful outside the laboratory. In the future, $D V P$ could be used to mitigate (or even prevent) the cybersickness experienced when using commercial HMD VR apps. For example, real-time estimates of the user's DVP calculated during the exposure could be used to provide warnings whenever he/she makes potentially provocative head movements. Alternatively, developers could script the storyline of the HMD VR gameplay/experience to intermix more and less provocative periods of $D V P$ - with the latter, calmer periods either being used to prevent the user from reaching the threshold for sickness or allowing him/her time to recover from any sickness that has been triggered.

\section{Will cybersickness disappear as baseline system lags are reduced?}

It is now possible to achieve an effective display lag of $\sim 3-$ $4 \mathrm{~ms}$ in HMD VR. However, some participants still report sickness symptoms even under these minimal lag conditions (see Figures 2A-C). Studies testing recent commercial VR games also continue to find quite high rates of sickness in their users even when modern HMD systems are used (e.g., Yildirim, 2019a; Teixeira and Palmisano, 2020). As noted earlier, provocative DVP can still be produced when baseline lag is artificially reduced to very low levels. We believe that latency spikes are the most likely explanation for cybersickness in this situation. According to our hypothesis, the change in DVP produced by brief latency spikes should be sufficient to trigger sickness in susceptible users. Consistent with this proposal, latency spikes have been shown to exacerbate cybersickness in HMD VR, even when participants do not notice them (Stauffert et al., 2018). Thus, researchers need to better understand the DVP generated by this unpredictable display lag. However, even when lag is adequately compensated, other errors in tracking the moving viewpoint could also produce provocative DVP. For example, ocular parallax produces small shifts in the effective vantage point during large eye movements (Mapp and Ono, 1986; Bingham, 1993). The high magnification of the near-eye displays used in HMDs can amplify these effects, but this DVP is not typically modeled when rendering HMD displays (Kudo and Ohnishi, 2000; Jones et al., 2015; Konrad et al., 2020). The above considerations therefore suggest that software techniques which artificially reduce the effective display lag (e.g., ATW) will not be a complete solution to cybersickness in HMD VR.

\section{Implications for HMD based augmented reality (AR)}

While this paper has focused on the cybersickness experienced in HMD VR, display lag can also be a problem for HMD AR (Bajura and Neumann, 1995; Yokokohji et al., 2000). For example, with video-see through HMDs, delay in the video camera feed can introduce an additional source of display lag. When the user makes a head-movement, their delayed camera images will often not match the virtual scene content. Different parts of the visual display will appear to be moving at different speeds (depending on whether they are real or virtual), promoting perceived scene instability and further increasing the likelihood of cybersickness. In a recent study, Freiwald et al. (2018) showed that cybersickness in HMD AR could be considerably reduced by imposing an additional constant delay to their HMD's tracking system so that it matched the latency of the video stream. By minimizing the discrepancies between visual real world and virtual scene content motion, this "CamWarp" technique should have reduced the users' perceived scene instability. However, it should have had little effect on their virtual head pose, as this would have been determined by the motion of their visual background. Since CamWarp only delayed the virtual foreground scene content, the user's DVP should have been largely unaffected by this technique. This intriguing finding suggests that perceived scene instability 
also plays an important role in cybersickness in HMD-AR (i.e., in addition to the user's DVP).

\section{CONCLUSIONS}

There have been substantial improvements to HMD hardware and software over the last decade. However, we are still far from fully understanding the cause of cybersickness and how it can be mitigated. This understanding is critical for HMD VR to reach its full potential and make access to the technology a preferred option for future ways of working (e.g., in education, training and health). In this paper, we present a new hypothesis for understanding cybersickness in HMD VR, based on Differences in the user's Virtual and Physical head pose (or DVP). We propose that DVP is the primary cause of cybersickness in HMD VR (not excessive eye-movements, or increases in postural instability, or conflicts involving vection, subjective verticals and rest frames). Our hypothesis is supported by empirical evidence that DVP can be used to predict the effects of display lag and head speed on cybersickness severity. Of the measures examined thus far, the mean, peak and standard deviation of the DVP appear to be the best predictors of cybersickness. However, we acknowledge that the current data are limited. Using DVP researchers and developers should be able to objectively estimate the likelihood and severity of cybersickness in virtual environments viewed using HMDs. It is hoped that in the future, estimates of this DVP could also be used to mitigate (or even prevent) the cybersickness experienced when using commercial HMD VR apps.

\section{DATA AVAILABILITY STATEMENT}

The data analyzed in this study is subject to the following licenses/restrictions: These datasets were published in

\section{REFERENCES}

Akiduki, H., Nishiike, S., Watanabe, H., Matsuoka, K., Kubo, T., and Takeda, N. (2003). Visual-vestibular conflict induced by virtual reality in humans. Neurosci. Lett. 340, 197-200. doi: 10.1016/S0304-3940(03)00098-3

Allison, R. S., Harris, L. R., Jenkin, M., Jasiobedzka, U., and Zacher, J. E. (2001). "Tolerance of temporal delay in virtual environments," in Proceedings of IEEE Virtual Reality (Yokohama: IEEE), 247-254.

Allison, R. S., Howard, I. P., and Zacher, J. E. (1999). Effect of field size, head motion and rotational velocity on roll vection and illusory self-tilt in a tumbling room. Perception 28, 299-306. doi: 10.1068/p2891

Andersen, D., and Popescu, V. (2018). "HMD-guided image-based modeling and rendering of indoor scenes," in Virtual Reality and Augmented Reality. EuroVR 2018. Lecture Notes in Computer Science, Vol. 11162, eds P. Bourdot, S. Cobb, V. Interrante, H. Kato, D. Stricker (Cham: Springer). 73-93. doi: 10.1007/978-3-030-01790-3_5

Apt, L., Isenberg, S., and Gaffney, W. L. (1973). The oculocardiac reflex in strabismus surgery. Am. J. Ophthalmol. 76, 533-536. doi: 10.1016/0002-9394(73)90743-5

Apthorp, D., Nagle, F., and Palmisano, S. (2014). Chaos in balance: nonlinear measures of postural control predict individual variations in visual illusions of motion. PLOS ONE 9:e113897. doi: 10.1371/journal.pone. 0113897 the journal Computers in Human Behavior and the proceedings of VRST 2019. Requests to access these datasets should be directed to juno.kim@unsw.edu.au and stephenp@uow.edu.au.

\section{ETHICS STATEMENT}

The studies involving human participants were reviewed and approved by the University of Wollongong and University of New South Wales Human Research Ethics Committees. The patients/participants provided their written informed consent to participate in this study.

\section{AUTHOR CONTRIBUTIONS}

SP: critical review of the literature. SP and JK: development of the hypothesis. SP, RA, and JK: theoretical analysis and wrote the paper. All authors: contributed to the article and approved the submitted version.

\section{FUNDING}

This paper was supported by a UOW study leave assistance grant to SP (including open access publication fees), an NUW Alliance grant to $\mathrm{SP}$ and $\mathrm{JK}$, and an Australian Research Council (ARC) Future Fellowship awarded to JK (FT140100535).

\section{SUPPLEMENTARY MATERIAL}

The Supplementary Material for this article can be found online at: https://www.frontiersin.org/articles/10.3389/frvir. 2020.587698/full\#supplementary-material

Arcioni, B., Palmisano, S., Apthorp, D., and Kim, J. (2019). Postural stability predicts the likelihood of cybersickness in active HMDbased virtual reality. Displays 58, 3-11. doi: 10.1016/j.displa.2018. 07.001

Azuma, R. T. (1997). A survey of augmented reality. Presence 6, 560-571. doi: 10.1162/pres.1997.6.4.355

Bajura, M., and Neumann, U. (1995). Dynamic registration correction in videobased augmented reality systems. IEEE Comput. Graph. Appl. 15, 52-60. doi: $10.1109 / 38.403828$

Baltzley, D. R., Kennedy, R. S., Berbaum, K. S., Lilienthal, M. G., and Gower, D. W. (1989). The time course of postflight simulator sickness symptoms. Aviat. Space Environ. Med. 60, 1043-1048.

Bernardo, A. (2017). Virtual reality and simulation in neurosurgical training. World Neurosurg. 106, 1015-1029. doi: 10.1016/j.wneu.2017.06.140

Bingham, G. P. (1993). Optical flow from eye movement with head immobilized: "Ocular occlusion" beyond the nose. Vision Res. 33, 777-789. doi: 10.1016/0042-6989(93)90197-5

Biocca, F. (1992). Will simulation sickness slow down the diffusion of virtual environment technology? Presence 1, 334-343. doi: 10.1162/pres.1992.1.3.334

Blascovich, J., Loomis, J., Beall, A. C., Swinth, K. R., Hoyt, C. L., and Bailenson, J. N. (2002). Immersive virtual environment technology as a methodological tool for social psychology. Psychol. Inq. 13, 103-124. doi: 10.1207/S15327965PLI1302_01 
Bles, W., Bos, J. E., De Graaf, B., Groen, E., and Wertheim, A. H. (1998). Motion sickness: only one provocative conflict? Brain Res. Bull. 47, 481-487. doi: 10.1016/S0361-9230(98)00115-4

Bles, W., Bos, J. E., and Kruit, H. (2000). Motion sickness. Curr. Opin. Neurol. 13, 19-25. doi: 10.1097/00019052-200002000-00005

Bonato, F., Bubka, A., and Alfieri, L. (2004). Display color affects motion sickness symptoms in an optokinetic drum. Aviat. Space Environ. Med. 75, 306-311.

Bonato, F., Bubka, A., and Palmisano, S. (2009). Combined pitch and roll and cybersickness in a virtual environment. Aviat. Space Environ. Med. 80, 941-945. doi: 10.3357/ASEM.2394.2009

Bonato, F., Bubka, A., Palmisano, S., Phillip, D., and Moreno, G. (2008). Vection change exacerbates simulator sickness in virtual environments. Presence 17, 283-292. doi: 10.1162/pres.17.3.283

Bonato, F., Bubka, A., and Story, M. (2005). Rotation direction change hastens motion sickness onset in an optokinetic drum. Aviat. Space Environ. Med. $76,823-827$.

Bonnet, C. T., Faugloire, E., Riley, M. A., Bardy, B. G., and Stoffregen, T. A. (2006). Motion sickness preceded by unstable displacements of the center of pressure. Hum. Mov. Sci. 25, 800-820. doi: 10.1016/j.humov.2006.03.001

Bos, J. E., and Bles, W. (1998). Modelling motion sickness and subjective vertical mismatch detailed for vertical motions. Brain Res. Bull. 47, 537-542. doi: 10.1016/S0361-9230(98)00088-4

Bos, J. E., and Bles, W. (2002). Theoretical considerations on canalotolith interaction and an observer model. Biol. Cybernetics 86, 191-207. doi: 10.1007/s00422-001-0289-7

Bos, J. E., Bles, W., and Groen, E. L. (2008). A theory on visually induced motion sickness. Displays 29, 47-57. doi: 10.1016/j.displa.2007.09.002

Boyd, D. (2014). Is the Oculus Rift Sexist? Available online at: http://qz.com/ 192874/is-the-oculus-rift-designed-to-be-sexist/ (accessed July 14, 2020).

Bronstein, A. M., and Gresty, M. A. (1988). Short latency compensatory eye movement responses to transient linear head acceleration: a specific function of the otolith-ocular reflex. Exp. Brain Res. 71, 406-410. doi: 10.1007/BF00247500

Bubka, A., and Bonato, F. (2003). Optokinetic drum tilt hastens the onset of vection-induced motion sickness. Aviat. Space Environ. Med. 74, 315-319.

Bubka, A., Bonato, F., and Palmisano, S. (2007). Expanding and contracting optic flow patterns and simulator sickness. Aviat. Space Environ. Med. 78, 383-386.

Cao, S., Nandakumar, K., Babu, R., and Thompson, B. (2019). Game play in virtual reality driving simulation involving head-mounted display and comparison to desktop display. Virt. Reality 24, 503-513. doi: 10.1007/s10055-019-00412-x

Cao, Z., Jerald, J., and Kopper, R. (2018)."Visually-induced motion sickness reduction via static and dynamic rest frames," in Proceedings of IEEE Conference on Virtual Reality and 3D User Interfaces (Reutlingen: IEEE), 105-112. doi: 10.1109/VR.2018.8446210

Caserman, P., Martinussen, M., and Göbel, S. (2019). "Effects of end-toend latency on user experience and performance in immersive virtual reality applications," in Proceedings of the Joint International Conference on Entertainment Computing and Serious Games (Arequipa: Springer), 57-69. doi: 10.1007/978-3-030-34644-7_5

Chang, C. H., Pan, W. W., Chen, F. C., and Stoffregen, T. A. (2013). Console video games, postural activity, and motion sickness during passive restraint. Exp. Brain Res. 229, 235-242. doi: 10.1007/s00221-013-3609-y

Chang, C. H., Pan, W. W., Tseng, L. Y., and Stoffregen, T. A. (2012). Postural activity and motion sickness during video game play in children and adults. Exp. Brain Res. 217, 299-309. doi: 10.1007/s00221-011-2993-4

Chang, E., Hwang, I., Jeon, H., Chun, Y., Kim, H. T., and Park, C. (2013). "Effects of rest frames on cybersickness and oscillatory brain activity," in Proceedings of the International Winter Workshop on Brain-Computer Interface (Gangwo: IEEE), 62-64. doi: 10.1109/IWW-BCI.2013.6506631

Chen, D. J., Chow, E. H., and So, R. H. (2011). The relationship between spatial velocity, vection, and visually induced motion sickness: an experimental study. i-Perception 2:415. doi: 10.1068/ic415

Chen, E., Luu, W., Chen, R., Rafik, A., Ryu, Y., Zangerl, B., et al. (2020). Virtual reality improves clinical assessment of the optic nerve. Front. Virt. Real. 1:4. doi: 10.3389 /frvir.2020.00004

Chen, W., Chao, J. G., Wang, J. K., Chen, X. W., and Tan, C. (2016). Subjective vertical conflict theory and space motion sickness. Aerosp Med. Hum. Perform. 87, 128-136. doi: 10.3357/AMHP.4327.2016
Cheung, B. S. K., Howard, I. P., and Money, K. E. (1991). Visually induced sickness in normal and bilaterally labyrinthine-defective subjects. Aviat. Space Environ. Med. 62, 527-531.

Ch'ng, E. (2009). Experiential archaeology: is virtual time travel possible? J. Cult. Herit. 10, 458-470. doi: 10.1016/j.culher.2009.02.001

Claremont, C. A. (1931). The psychology of seasickness. Psyche 11, 86-90.

Clark, B., and Stewart, J. D. (1970). Thresholds for the perception of angular acceleration about the three major body axes. Acta Otolaryngol. 69, 231-238. doi: $10.3109 / 00016487009123358$

Clifton, J., and Palmisano, S. (2019). Effects of steering locomotion and teleporting on cybersickness and presence in HMD-based virtual reality. Virtual Real. 24, 453-468. doi: 10.1007/s10055-019-00407-8

Cobb, S. V. G. (1999). Measurement of postural stability before and after immersion in a virtual environment. Appl. Ergon. 30, 47-57. doi: 10.1016/S0003-6870(98)00038-6

Cobb, S. V. G., and Nichols, S. C. (1998). Static posture tests for the assessment of postural instability after virtual environment use. Brain Res. Bull. 47, 459-464. doi: 10.1016/S0361-9230(98)00104-X

Collewijn, H., and Smeets, J. B. (2000). Early components of the human vestibuloocular response to head rotation: latency and gain. J. Neurophysiol. 84, 376-389. doi: $10.1152 /$ jn.2000.84.1.376

Cook, H. E., Hassebrock, J. A., and Smart, L. J. Jr. (2018). Responding to other people's posture: visually induced motion sickness from naturally generated optic flow. Front. Psychol. 9:1901. doi: 10.3389/fpsyg.2018.01901

Cummings, J. J., and Bailenson, J. N. (2016). How immersive is enough? A metaanalysis of the effect of immersive technology on user presence. Media Psychol. 19, 272-309. doi: 10.1080/15213269.2015.1015740

Curry, C., Li, R., Peterson, N., and Stoffregen, T. A. (2020). Cybersickness in virtual reality head-mounted displays: examining the influence of sex differences and vehicle control. Int. J. Hum. Comput. Interact. 36, 1161-1167. doi: 10.1080/10447318.2020.1726108

Davis, S., Nesbitt, K., and Nalivaiko, E. (2014). "A systematic review of cybersickness," in Proceedings of the Conference on Interactive Entertainment (Newcastle, NSW: ACM), 1-9. doi: 10.1145/2677758.2677780

de Graaf, B., Bles, W., and Bos, J. E. (1998). Roll motion stimuli: Sensory conflict, perceptual weighting and motion sickness. Brain Res. Bull. 47, 489-495. doi: 10.1016/S0361-9230(98)00116-6

Dennison, M. S., and D'Zmura, M. (2017). Cybersickness without the wobble: experimental results speak against postural instability theory. Appl. Ergon. 58, 215-223. doi: 10.1016/j.apergo.2016.06.014

Dennison, M. S., Wisti, A. Z., and D'Zmura, M. (2016). Use of physiological signals to predict cybersickness. Displays 44, 42-52. doi: 10.1016/j.displa.2016.07.002

Diels, C., Ukai, K., and Howarth, P. A. (2007). Visually induced motion sickness with radial displays: effects of gaze angle and fixation. Aviat. Space Environ. Med. 78, 659-665.

DiZio, P., and Lackner, J. R. (1997). "Circumventing side effects of immersive virtual environments," in Advances in Human Factors/Ergonomics: Vol. 21 b. Design of Computing Systems: Social and Ergonomic Considerations, eds G. Salvendy, M. J. Smith, G. Salvendy, and R. J. Koubek (Amsterdam: Elsevier), 893-896.

Dong, X., Yoshida, K., and Stoffregen, T. A. (2011). Control of a virtual vehicle influences postural activity and motion sickness. J. Exp. Psychol. Appl. 17, 128-138. doi: 10.1037/a0024097

Draper, M. H., Viirre, E. S., Furness, T. A., and Gawron, V. J. (2001). Effects of image scale and system time delay on simulator sickness within head-coupled virtual environments. Hum. Factors 43, 129-146. doi: 10.1518/001872001775992552

Duh, H. B. L., Parker, D. E., and Furness, T. A. (2004). An independent visual background reduced simulator sickness in a driving simulator. Presence 13, 578-588. doi: 10.1162/1054746042545283

Ebenholtz, S. M. (1992). Motion sickness and oculomotor systems in virtual environments. Presence 1, 302-305. doi: 10.1162/pres.1992.1.3.302

Ebenholtz, S. M., Cohen, M. M., and Linder, B. J. (1994). The possible role of nystagmus in motion sickness: a hypothesis. Aviat. Space Environ. Med. 65, 1032-1035.

Elliman, J., Loizou, M., and Loizides, F. (2016). "Virtual reality simulation training for student nurse education," in Proceedings of the 8th International Conference 
on Games and Virtual Worlds for Serious Applications (Barcelona: IEEE), 1-2. doi: 10.1109/VS-GAMES.2016.7590377

Ellis, S. R., Mania, K., Adelstein, B. D., and Hill, M. I. (2004). "Generalizeability of latency detection in a variety of virtual environments," in Proceedings of the Human Factors and Ergonomics Society Annual Meeting (Los Angeles, CA: SAGE), 48, 2632-2636. doi: 10.1177/154193120404802306

Eubanks, J. C., Somareddy, V., McMahan, R. P., and Lopez, A. A. (2016). "Fullbody portable virtual reality for personal protective equipment training," in Proceedings of the International Conference on Virtual, Augmented and Mixed Reality (Toronto, ON: HCI), 490-501. doi: 10.1007/978-3-319-39907-2_47

Feng, J., Kim, J., Luu, W., and Palmisano, S. (2019). “Method for estimating display lag in the Oculus Rift S and CV1" in Proceedings of SIGGRAPH Asia (Brisbane, QLD: SIGGRAPH) 39, 1-2. doi: 10.1145/3355056.3364590

Flanagan, M. B., May, J. G., and Dobie, T. G. (2002). Optokinetic nystagmus, vection, and motion sickness. Aviat. Space Environ. Med. 73, 1067-1073.

Flanagan, M. B., May, J. G., and Dobie, T. G. (2004). The role of vection, eye movements and postural instability in the etiology of motion sickness. J. Vestib. Res. 14, 335-346.

Frank, L. H., Casali, J. G., and Wierwille, W. W. (1988). Effects of visual display and motion system delays on operator performance and uneasiness in a driving simulator. Hum. Factors 30, 201-217. doi: 10.1177/001872088803000207

Freiwald, J. P., Katzakis, N., and Steinicke, F. (2018). "Camera time warp: compensating latency in video see-through head-mounted-displays for reduced cybersickness effects," in Proceedings of the 24th ACM Symposium on Virtual Reality Software and Technology (Tokyo: ACM), 1-7. doi: 10.1145/3281505.3281521

Gavgani, A., Walker, F. R., Hodgson, D. M., and Nalivaiko, E. (2018). A comparative study of cybersickness during exposure to virtual reality and "classic" motion sickness: are they different? J. Appl. Physiol. 125, 1670-1680. doi: 10.1152/japplphysiol.00338.2018

Gavgani, A. M., Hodgson, D. M., and Nalivaiko, E. (2017b). Effects of visual flow direction on signs and symptoms of cybersickness. PLOS ONE 12:e1082790. doi: 10.1371/journal.pone.0182790

Gavgani, A. M., Nesbitt, K. V., Blackmore, K. L., and Nalivaiko, E. (2017a). Profiling subjective symptoms and autonomic changes associated with cybersickness. Auton. Neurosci. 203, 41-50. doi: 10.1016/j.autneu.2016.12.004

Gibson, J. J. (1950). The Perception of the Visual World. Boston, MA: Houghton Mifflin.

Golding, J. F. (2016). "Motion sickness," in Handbook of Clinical Neurology, Vol. 137. 3rd Series Neuro-Otology, eds J. M. Furman and T. Lempert (Amsterdam: Elsevier), 371-390.

Golding, J. F., Doolan, K., Acharya, A., Tribak, M., and Gresty, M. A. (2012). Cognitive cues and visually induced motion sickness. Aviat. Space Environ. Med. 83, 477-482. doi: 10.3357/ASEM.3095.2012

Golding, J. F., Mueller, A. G., and Gresty, M. A. (2001). A motion sickness maximum around the $0.2 \mathrm{~Hz}$ frequency range of horizontal translational oscillation. Aviat. Space Environ. Med. 72, 188-192.

Gonizzi Barsanti, S., Caruso, G., Micoli, L. L., Covarrubias Rodriguez, M., and Guidi, G. (2015). "3D visualization of cultural heritage artefacts with virtual reality devices," in Proceedings of the 25th International CIPA Symposium (Taipei: Copernicus Gesellschaft $\mathrm{mbH}$ ).

Grabherr, L., Nicoucar, K., Mast, F. W., and Merfeld, D. M. (2008). Vestibular thresholds for yaw rotation about an earth-vertical axis as a function of frequency. Exp. Brain Res. 186, 677-681. doi: 10.1007/s00221-008-1350-8

Grabowski, A., and Jankowski, J. (2015). Virtual reality-based pilot training for underground coal miners. Saf. Sci. 72, 310-314. doi: 10.1016/j.ssci.2014.09.017

Guedry, F. E. (1974). "Psychophysics of vestibular sensation," in Handbook of Sensory Physiology, Vol VI. ed H. H. Kornhuber (New York, NY: Springer), 3-154. doi: 10.1007/978-3-642-65920-1_1

Guedry, F. E. (1991). "Motion sickness and its relation to some forms of spatial orientation: Mechanisms and theory," in Motion Sickness: Significance in Aerospace Operations and Prophylaxis, Various eds (Neuilly sur Seine: North Atlantic Treaty Organization, Advisory Group for Aerospace Research and Development), 1-30.

Guedry, F. E., and Montague, E. K. (1961). Quantitative evaluation of the vestibular Coriolis reaction. Aerosp. Med. 32, 487-500.

Guo, C. C., Chen, D. J., Wei, I. Y., So, R. H., and Cheung, R. T. (2017). Correlations between individual susceptibility to visually induced motion sickness and decaying time constant of after-nystagmus. Appl. Ergon. 63, 1-8. doi: 10.1016/j.apergo.2017.03.011

Guo, C. T., So, R. H. Y., Kwok, K., and Cheung, R. (2012). "Effects of foveal retinal slip on visually induced motion sickness under controlled of eye motions," in the Proceedings of the Annual Meeting of the Human Factors and Ergonomics Society (Boston, MA: HFES), 22-26.

Gupta, V. K. (2005). Motion sickness is linked to nystagmus-related trigeminal brain stem input: a new hypothesis. Med. Hypotheses. 64, 1177-1181. doi: 10.1016/j.mehy.2004.11.031

Han, K. S., and Cho, J. H. (2019). The effect of presence and engagement in HMD (Head Mounted Display) virtual reality advertising on attitude and memory. J. Korea Convergence Soc. 10, 139-146. doi: 10.15207/JKCS.2019.10.6.139

Hettinger, L. J., Berbaum, K. S., Kennedy, R. S., Dunlap, W. P., and Nolan, M. D. (1990). Vection and simulator sickness. Milit. Psychol. 2, 171-181. doi: 10.1207/s15327876mp0203_4

Hill, K. J., and Howarth, P. A. (2000). Habituation to the side effects of immersion in a virtual environment. Displays 21, 25-30. doi: 10.1016/S0141-9382(00)00029-9

Hogue, J., Anderson, F., Pelz, C., Allen, R., Markhamn, S., and Harmsen, A. (1999). "Enhanced parachute simulation training and planning," in Proceedings of the 15th Aerodynamic Decelerator Systems Technology Conference (Toulouse: ARC), 1724. doi: 10.2514/6.1999-1724

Howard, I. P. (1982). Human Visual Orientation. Chichester: John Wiley.

Howard, I. P., and Childerson, L. (1994). The contribution of motion, the visual frame, and visual polarity to sensations of body tilt. Perception 23, 753-762. doi: $10.1068 / \mathrm{p} 230753$

Howard, I. P., and Hu, G. (2001). Visually induced reorientation illusions. Perception 30, 583-600. doi: 10.1068/p3106

Howarth, P. A., and Costello, P. J. (1997). The occurrence of virtual simulation sickness symptoms when an HMD was used as a personal viewing system. Displays 18, 107-116. doi: 10.1016/S0141-9382(97)00011-5

Howarth, P. A., and Finch, M. (1999). The nauseogenicity of two methods of navigating within a virtual environment. Appl. Ergon. 30, 39-45. doi: 10.1016/S0003-6870(98)00041-6

Howarth, P. A., and Hodder, S. G. (2008). Characteristics of habituation to motion in a virtual environment. Displays 29, 117-123. doi: 10.1016/j.displa.2007.09.009

$\mathrm{Hu}, \mathrm{S}$., and Stern, R. M. (1998). Optokinetic nystagmus correlates with severity of vection-induced motion sickness and gastric tachyarrhythmia. Aviat. Space Environ. Med. 69, 1162-1165.

Hu, S. Q., Davis, M. S., Klose, A. H., Zabinsky, E. M., Meux, S. P., Jacobsen, H. A., et al. (1997). Effects of spatial frequency of a vertically striped rotating drum on vection-induced motion sickness. Aviat. Space Environ. Med. 68, 306-311.

Jennings, S., Craig, G., Reid, L., and Kruk, R. (2000). “The effect of visual system time delay on helicopter control," in Proceedings of the Human Factors and Ergonomics Society Annual Meeting (Los Angeles, CA: SAGE), 44, 69-72. doi: 10.1177/154193120004401318

Jennings, S., Reid, L. D., Craig, G., and Kruk, R. V. (2004). Time delays in visually coupled systems during flight test and simulation. J. Aircr. 41, 1327-1335. doi: $10.2514 / 1.449$

Jensen, L., and Konradsen, F. (2018). A review of the use of virtual reality head-mounted displays in education and training. Educ. Inform. Technol. 23, 1515-1529. doi: 10.1007/s10639-017-9676-0

Ji, J. T., So, R. H., and Cheung, R. T. (2009). Isolating the effects of vection and optokinetic nystagmus on optokinetic rotation-induced motion sickness. Hum. Factors 51, 739-751. doi: 10.1177/0018720809349708

Jones, J. A., Dukes, L. C., Krum, D. M., Bolas, M. T., and Hodges, L. F. (2015). "Correction of geometric distortions and the impact of eye position in virtual reality displays," in 2015 International Conference on Collaboration Technologies and Systems (CTS) (Atlanta, GA), 77-83. doi: 10.1109/CTS.2015.7210403

Karmali, F., Lim, K., and Merfeld, D. M. (2014). Visual and vestibular perceptual thresholds each demonstrate better precision at specific frequencies and also exhibit optimal integration. J. Neurophysiol. 111, 2393-2403. doi: 10.1152/jn.00332.2013

Kennedy, R. S., Drexler, J., and Kennedy, R. C. (2010). Research in visually induced motion sickness. Appl. Ergon. 41, 494-503. doi: 10.1016/j.apergo.2009.11.006

Kennedy, R. S., Graybiel, A., McDonough, R. C., and Beckwith, F. D. (1968). Symptomatology under storm conditions in the North Atlantic in control 
subjects and in persons with bilateral labyrinthine defects. Acta Otolaryngol. 66, 533-540. doi: 10.3109/00016486809126317

Kennedy, R. S., Hettinger, L. J., and Lilienthal, M. G. (1990). "Simulator sickness," in Motion and Space Sickness, ed G. H. Crampton (Boca Raton, FL: CRC Press), 317-342.

Kennedy, R. S., Jones, M. B., Lilienthal, M. G., and Harm, D. L. (1994). Profile analysis of after-effects experienced during exposure to several virtual reality environments. AGARD-CP-541. Virt. Interf. Res. Appl. 2, 1-9.

Kennedy, R. S., Lane, N. E., Berbaum, K. S., and Lilienthal, M. G. (1993). Simulator sickness questionnaire: an enhanced method for quantifying simulator sickness. Int. J. Aviat. Psychol. 3, 203-220. doi: 10.1207/s15327108ijap0303_3

Kennedy, R. S., and Lilienthal, M. G. (1994). "Measurement and control of motion sickness after-effects from immersion in virtual reality," in Proceedings of Virtual Reality and Medicine: The Cutting Edge (New York, NY: SIG-Advanced Applications), 111-119.

Kennedy, R. S., and Stanney, K. M. (1996). Postural instability induced by virtual reality exposure: development of a certification protocol. Int. J. Hum. Comput. Interact. 8, 25-47. doi: 10.1080/10447319609526139

Keshavarz, B., and Hecht, H. (2011a). Axis rotation and visually induced motion sickness: the role of combined roll, pitch, and yaw motion. Aviat. Space Environ. Med. 82, 1023-1029. doi: 10.3357/ASEM.3078.2011

Keshavarz, B., and Hecht, H. (2011b). Validating an efficient method to quantify motion sickness. Hum. Factors 53, 415-426. doi: 10.1177/0018720811403736

Keshavarz, B., Hecht, H., and Lawson, B. D. (2014). "Visually induced motion sickness: causes, characteristics, and countermeasures," in Handbook of Virtual Environments: Design, Implementation, and Applications, eds K. S. Hale and K. M. Stanney (Boca Raton, FL: CRC Press), 652-703. doi: 10.1201/b17360-32

Keshavarz, B., Novak, A. C., Hettinger, L. J., Stoffregen, T. A., and Campos, J. L. (2017). Passive restraint reduces visually induced motion sickness in older adults. J. Exp. Psychol. 23, 85-99. doi: 10.1037/xap0000107

Keshavarz, B., Riecke, B. E., Hettinger, L. J., and Campos, J. L. (2015). Vection and visually induced motion sickness: how are they related? Front. Psychol. 6:472. doi: 10.3389/fpsyg.2015.00472

Khor, W. S., Baker, B., Amin, K., Chan, A., Patel, K., and Wong, J. (2016). Augmented and virtual reality in surgery-the digital surgical environment: applications, limitations and legal pitfalls. Ann. Transl. Med. 4:23. doi: 10.21037/atm.2016.12.23

Kim, J., Chung, C. Y., Nakamura, S., Palmisano, S., and Khuu, S. K. (2015). The Oculus Rift: a cost-effective tool for studying visual-vestibular interactions in self-motion perception. Front. Psychol. 6:248. doi: 10.3389/fpsyg.2015.00248

Kim, J., Luu, W., and Palmisano, S. (2020). Multisensory integration and the experience of scene instability, presence and cybersickness in virtual environments. Comput. Hum. Behav. 113:106484 doi: $10.1016 /$ j.chb.2020.106484

Kim, K., Rosenthal, M. Z., Zielinski, D. J., and Brady, R. (2014). Effects of virtual environment platforms on emotional responses. Comput. Methods Prog. Biomed. 113, 882-893. doi: 10.1016/j.cmpb.2013.12.024

Kingdon, K. S., Stanney, K. M., and Kennedy, R. S. (2001). "Extreme responses to virtual environment exposure," in Proceedings of the Human Factors and Ergonomics Society Annual Meeting (Los Angeles, CA: SAGE).

Kinsella, A., Mattfeld, R., Muth, E., and Hoover, A. (2016). Frequency, not amplitude, of latency affects subjective sickness in a head-mounted display. Aerosp. Med. Hum. Perform. 87, 604-609. doi: 10.3357/AMHP.4351.2016

Kiruluta, A., Eizenman, M., and Pasupathy, S. (1997). Predictive head movement tracking using a Kalman filter. IEEE Trans. Syst. Man Cybernetics Part B. 27, 326-331. doi: 10.1109/3477.558841

Konrad, R., Angelopoulos, A., and Wetzstein, G. (2020). Gaze-contingent ocular parallax rendering for virtual reality. ACM Trans. Graphics 39:10. doi: $10.1145 / 3361330$

Koslucher, F., Haaland, E., and Stoffregen, T. A. (2016). Sex differences in visual performance and postural sway precede sex differences in visually induced motion sickness. Exp. Brain Res. 234, 313-322. doi: 10.1007/s00221-015-4462-y

Koslucher, F. C., Haaland, E., Malsch, A., Webeler, J., and Stoffregen, T. A. (2015). Sex differences in the incidence of motion sickness induced by linear visual oscillation. Aviat. Med. Hum. Perform. 86, 787-793. doi: 10.3357/AMHP.4243.2015

Kudo, H., and Ohnishi, N. (2000). "Effect of the sight line shift when a headmounted display is used," in Proceedings of the 22nd Annual International
Conference of the IEEE Engineering in Medicine and Biology Society (Cat. No. 00CH37143). (Chicago, IL).

Kuiper, O. X., Bos, J. E., and Diels, C. (2019). Vection does not necessitate visually induced motion sickness. Displays 58, 82-87. doi: 10.1016/j.displa.2018. 10.001

Lackner, J. R., and DiZio, P. (2006). Space motion sickness. Exp. Brain Res. 175, 377-399. doi: 10.1007/s00221-006-0697-y

Lackner, J. R., and DiZio, P. (2020). Velocity storage: its multiple roles. J. Neurophysiol.123, 1206-1215. doi: 10.1152/jn.00139.2019

LaViola, J. J. Jr. (2000). A discussion of cybersickness in virtual environments. ACM SIGCHI Bull. 32, 47-56. doi: 10.1145/333329.333344

Lawson, B. (2014). "Motion sickness symptomatology and origins," in Handbook of Virtual Environments: Design, Implementation, and Applications, eds K. S. Hale and K. M. Stanney (Boca Raton, FL: CRC Press), 532-587. doi: 10.1201/b17360-29

Lawson, B. D. (2005). "Exploiting the illusion of self-motion (vection) to achieve a feeling of 'virtual acceleration' in an immersive display," in Proceedings of the 11th International Conference on Human-Computer Interaction (Las Vegas, NV: HCI), 1-10.

Lee, D. N., and Lishman, J. R. (1975). Visual proprioceptive control of stance. J. Hum. Move. Stud. 1, 87-95.

Lewis, T. (2015). When Will Virtual-Reality Headsets Stop Making People Sick? Available online at: http://www.livescience.com/50129-virtual-realitynauseasickness.html (accessed July 14, 2020).

Lishman, J. R., and Lee, D. N. (1973). The autonomy of visual kinaesthesis Perception 2, 287-294. doi: 10.1068/p020287

MacNeilage, P. R., Turner, A. H., and Angelaki, D. E. (2010). Canalotolith interactions and detection thresholds of linear and angular components during curved-path self-motion. J. Neurophysiol. 104, 765-773. doi: 10.1152/jn.01067.2009

Mania, K., Adelstein, B. D., Ellis, S. R., and Hill, M. I. (2004). "Perceptual sensitivity to head tracking latency in virtual environments with varying degrees of scene complexity," in Proceedings of the ACM Siggraph Symposium on Applied Perception in Graphics and Visualization (New York, NY: ACM), 39-48. doi: 10.1145/1012551.1012559

Mapp, A. P., and Ono, H. (1986). The rhino-optical phenomenon: ocular parallax and the visible field beyond the nose. Vision Res. 26, 1163-1165. doi: 10.1016/0042-6989(86)90050-7

McCauley, M. E., and Sharkey, T. J. (1992). Cybersickness: perception of self-motion in virtual environments. Presence 1, 311-318. doi: 10.1162/pres.1992.1.3.311

Meehan, M., Razzaque, S., Whitton, M. C., and Brooks, F. P. (2003). "Effect of latency on presence in stressful virtual environments," in Proceedings of IEEE Virtual Reality (Los Angeles, CA: IEEE), 141-148. doi: 10.1109/VR.2003.1191132

Merhi, O., Faugloire, E., Flanagan, M., and Stoffregen, T. A. (2007). Motion sickness, console video games, and head mounted displays. Hum. Fact. 49, 920-934 doi: 10.1518/001872007X230262

Min, Y. K., Chung, S. C., You, J. H., Yi, J. H., Lee, B., Tack, G. R., et al. (2006). Young adult drivers' sensitivity to changes in speed and driving mode in a simple vehicle simulator. Percept. Mot. Skills 103, 197-209. doi: 10.2466/pms.103.1.197-209

Money, K. E., and Cheung, B. S. (1983). Another function of the inner ear: facilitation of the emetic response to poisons. Aviat. Space Environ. Med. 54, 208-211.

Money, K. E., and Wood, J. D. (1970). "Neural mechanisms underlying the symptomatology of motion sickness," in Proceedings of the Fourth Symposium on the Role of the Vestibular Organs in Space Exploration (Pensacola, FL: NASA SP-187), 35-44.

Moss, J. D., Austin, J., Salley, J., Coats, J., Williams, K., and Muth, E. R. (2011). The effects of display delay on simulator sickness. Displays, 32, 159-168. doi: 10.1016/j.displa.2011.05.010

Moss, J. D., and Muth, E. R. (2011). Characteristics of head-mounted displays and their effects on simulator sickness. Hum. Factors 53, 308-319. doi: 10.1177/0018720811405196

Mujber, T. S., Szecsi, T., and Hashmi, M. S. (2004). Virtual reality applications in manufacturing process simulation. J. Mater. Process. Technol. 155, 1834-1838. doi: 10.1016/j.jmatprotec.2004.04.401 
Munafo, J., Diedrick, M., and Stoffregen, T. A. (2017). The virtual reality headmounted display Oculus Rift induces motion sickness and is sexist in its effects. Exp. Brain Res. 235, 889-901. doi: 10.1007/s00221-016-4846-7

Nalivaiko, E., Rudd, J. A., and So, R. H. Y. (2014). Motion sickness, nausea and thermoregulation: the "toxic" hypothesis. Temperature 1, 164-171. doi: 10.4161/23328940.2014.982047

Nishiike, S., Okazaki, S., Watanabe, H., Akizuki, H., Imai, T., Uno, A., et al. (2013). The effect of visual-vestibulosomatosensory conflict induced by virtual reality on postural stability in humans. J. Med. Investig. 60, 236-239. doi: $10.2152 /$ jmi. 60.236

Nooij, S. A., Pretto, P., and Bülthoff, H. H. (2018). More vection means more velocity storage activity: a factor in visually induced motion sickness? Exp. Brain Res. 236, 3031-3041. doi: 10.1007/s00221-018-5340-1

Nooij, S. A., Pretto, P., Oberfeld, D., Hecht, H., and Bülthoff, H. H. (2017). Vection is the main contributor to motion sickness induced by visual yaw rotation: implications for conflict and eye movement theories. PLOS ONE 12:e0175305. doi: 10.1371/journal.pone. 0175305

Ohmi, M., Howard, I. P., and Landolt, J. P. (1987). Circular vection as a function of foreground-background relationships. Perception, 16, 17-22. doi: $10.1068 / \mathrm{p} 160017$

Oman, C. M. (1982). A heuristic mathematical model for the dynamics of sensory conflict and motion sickness hearing in classical musicians. Acta Otolaryngol. 94, 4-44. doi: 10.3109/00016488209108197

Oman, C. M. (1990). Motion sickness: a synthesis and evaluation of the sensory conflict theory. Can. J. Physiol. Pharmacol. 68, 294-303. doi: 10.1139/y90-044

Ortegon-Sarmiento, T., Uribe-Quevedo, A., Perez-Gutierrez, B., Vega-Medina, L., and Tibamoso, G. (2016). "Hand tracking and haptic-based jugular neonate central venous access procedure," in Proceedings of the International Conference on Virtual, Augmented and Mixed Reality (Toronto, ON: HCI), 521-531. doi: 10.1007/978-3-319-39907-2_50

Palmisano, S., Allison, R. S., Kim, J., and Bonato, F. (2011a). Simulated viewpoint jitter shakes sensory conflict accounts of self-motion perception. Seeing Perceiving 24, 173-200.

Palmisano, S., Allison, R. S., Schira, M. M., and Barry, R. J. (2015). Future challenges for vection research: definitions, functional significance, measures and neural bases. Front. Psychol. 6:193. doi: 10.3389/fpsyg.2015.00193

Palmisano, S., Arcioni, B., and Stapley, P. (2018). Predicting vection and visually induced motion sickness based on spontaneous postural activity. Exp. Brain Res. 236, 315-329. doi: 10.1007/s00221-017-5130-1

Palmisano, S., Bonato, F., Bubka, A., and Folder, J. (2007). Vertical display oscillation increases vection in depth and simulator sickness. Aviat. Space Environ. Med. 78, 951-956. doi: 10.3357/ASEM.2079.2007

Palmisano, S., Kim, J., Allison, R., and Bonato, F. (2011b). Simulated viewpoint jitter shakes sensory conflict accounts of vection. Seeing Perceiving 24, 173-200. doi: 10.1163/187847511X570817

Palmisano, S., Mursic, R., and Kim, J. (2017). Vection and cybersickness generated by head-and-display motion in the Oculus Rift. Displays 46, 1-8. doi: 10.1016/j.displa.2016.11.001

Palmisano, S., and Riecke, B. E. (2018). The search for instantaneous vection: an oscillating visual prime reduces vection onset latency. PLoS ONE 13:e0195886. doi: 10.1371/journal.pone.0195886

Palmisano, S., Szalla, L., and Kim, J. (2019). "Monocular viewing protects against cybersickness produced by head movements in the Oculus Rift," in Proceedings of the 25th ACM Symposium on Virtual Reality Software and Technology (Sydney, NSW: ACM), 1-2. doi: 10.1145/3359996.3364699

Patterson, R., Winterbottom, M. D., and Pierce, B. J. (2006). Perceptual issues in the use of head-mounted displays. Hum. Factors 48, 555-573. doi: $10.1518 / 001872006778606877$

Phan, V. T., and Choo, S. Y. (2010). A combination of augmented reality and google Earth's facilities for urban planning in idea stage. Int. J. Comput. Appl. 4, 26-34. doi: 10.5120/809-1149

Pot-Kolder, R., Veling, W., Counotte, J., and van Der Gaag, M. (2018). Anxiety partially mediates cybersickness symptoms in immersive virtual reality environments. Cyberpsychol. Behav. Soc. Netw. 21, 187-193. doi: 10.1089/cyber.2017.0082

Prothero, J. D., Draper, M. H., Furness, 3rd, T. A., Parker, D. E., and Wells, M. J. (1999). The use of an independent visual background to reduce simulator side-effects. Aviat. Space Environ. Med. 70(3 Pt 1), 277-283.
Prothero, J. D., and Parker, D. E. (2003). "A unified approach to presence and motion sickness," in Virtual and Adaptive Environments: Applications, Implications, and Human Performance Issues, eds L. J. Hettinger and M. W Hass (Mahwah, NJ: Lawrence Erlbaum), 47-66. doi: 10.1201/9781410608888.ch3

Reason, J. T. (1978). Motion sickness adaptation: a neural mismatch model. J. $R$. Soc. Med.71, 819-829. doi: 10.1177/014107687807101109

Reason, J. T., and Brand, J. J. (1975). Motion Sickness. New York, NY: Academic Press.

Rebenitsch, L., and Owen, C. (2016). Review on cybersickness in applications and visual displays. Virtual Real. 20, 101-125. doi: 10.1007/s10055-016-0285-9

Rebenitsch, L., and Owen, C. (2020). Estimating cybersickness from virtual reality applications. Virtual Real. doi: 10.1007/s10055-020-00446-6

Reed-Jones, R. J., Vallis, L. A., Reed-Jones, J. G., and Trick, L. M. (2008). The relationship between postural stability and virtual environment adaptation. Neurosci. Lett. 435, 204-209. doi: 10.1016/j.neulet.2008.02.047

Riccio, G. E., and Stoffregen, T. A. (1991). An ecological theory of motion sickness and postural instability. Ecol. Psychol. 3, 195-240. doi: 10.1207/s15326969eco0303_2

Riecke, B. E., and Jordan, J. D. (2015). Comparing the effectiveness of different displays in enhancing illusions of self-movement (vection). Front. Psychol. 6:713. doi: 10.3389/fpsyg.2015.00713

Risi, D., and Palmisano, S. (2019). Effects of postural stability, active control, exposure duration and repeated exposures on HMD induced cybersickness. Displays 60, 9-17. doi: 10.1016/j.displa.2019.08.003

Roditi, R. E., and Crane, B. T. (2012). Directional asymmetries and age effects in human self-motion perception. J. Assoc. Res. Otolaryngol. 13, 381-401. doi: $10.1007 / \mathrm{s} 10162-012-0318-3$

Rolnick, A., and Lubow, R. E. (1991). Why is the driver rarely motion sick? The role of controllability in motion sickness. Ergonomics 34, 867-879. doi: $10.1080 / 00140139108964831$

Schubert, T., Friedmann, F., and Regenbrecht, H. (2001). The experience of presence: factor analytic insights. Presence 10, 266-281. doi: 10.1162/105474601300343603

Seno, T., Sawai, K., Kanaya, H., Wakebe, T., Ogawa, M., Fujii, Y., et al. (2017). The oscillating potential model of vection. i-Perception 8, 1-24. doi: $10.1177 / 2041669517742176$

Sharples, S., Cobb, S., Moody, A., and Wilson, J. R. (2008). Virtual reality induced symptoms and effects (VRISE): comparison of head mounted display (HMD), desktop and projection display systems. Displays 29, 58-69. doi: 10.1016/j.displa.2007.09.005

Simons, R., and Melzer, J. E. (2003). "HMD-based training for the US Army's AVCATT-A collective aviation training simulator," in Proceedings of Helmetand Head-Mounted Displays VIII: Technologies and Applications (Orlando, FL: SPIE).

Skarbez, R., Brooks, F. J., and Whitton, M. C. (2017). A survey of presence and related concepts. ACM Comput Surv. 50:96. doi: 10.1145/3134301

Smart, L. J., Otten, E. W., Strang, A. J., Littman, E. M., and Cook, H. E. (2014). Influence of complexity and coupling of optic flow on visually induced motion sickness. Ecol. Psychol. 26, 301-324. doi: 10.1080/10407413.2014. 958029

Smart, L. J., Stoffregen, T. A., and Bardy, B. G. (2002). Visually induced motion sickness predicted by postural instability. Hum. Factors 44, 451-465. doi: $10.1518 / 0018720024497745$

Stanney, K., Salvendy, G., Deisinger, J., DiZio, P., Ellis, S., Ellision, J., et al. (1998b). Aftereffects and sense of presence in virtual environments: formulation of a research and development agenda. Report sponsored by the life sciences division at NASA headquarters. Int. J. Hum. Comput. Interact. 10, 135-187. doi: 10.1207/s15327590ijhc1002_3

Stanney, K. M., Mourant, R. R., and Kennedy, R. S. (1998a). Human factors issues in virtual environments: a review of the literature. Presence 7, 327-351. doi: $10.1162 / 105474698565767$

Stauffert, J. P., Niebling, F., and Latoschik, M. E. (2018). "Effects of latency jitter on simulator sickness in a search task," in IEEE Conference on Virtual Reality and 3D User Interfaces (VR) (Reutlingen: IEEE), 121-127. doi: 10.1109/VR.2018.8446195

Stern, R. M., Hu, S., Anderson, R. B., Leibowitz, H. W., and Koch, K. L. (1990). The effects of fixation and restricted visual field on vection-induced motion sickness. Aviat. Space Environ. Med. 61, 712-715. 
Stoffregen, T. A., Chen, Y. C., and Koslucher, F. C. (2014). Motion control, motion sickness, and the postural dynamics of mobile devices. Exp. Brain Res. 232, 1389-1397. doi: 10.1007/s00221-014-3859-3

Stoffregen, T. A., Faugloire, E., Yoshida, K., Flanagan, M. B., and Merhi, O. (2008). Motion sickness and postural sway in console video games. Hum. Factors 50, 322-331. doi: 10.1518/001872008X250755

Stoffregen, T. A., Hettinger, L. J., Haas, M. W., Roe, M. M., and Smart, L. J. (2000). Postural instability and motion sickness in a fixed-base flight simulator. Hum. Factors 42, 458-469. doi: 10.1518/001872000779698097

Stoffregen, T. A., and Riccio, G. E. (1991). An ecological critique of the sensory conflict theory of motion sickness. Ecol. Psychol. 3, 159-194. doi: 10.1207/s15326969eco0303_1

Stoffregen, T. A., and Smart, L. J. (1998). Postural instability precedes motion sickness. Brain Res. Bull. 47, 437-448. doi: 10.1016/S0361-9230(98)00102-6

Stoffregen, T. A., Yoshida, K., Villard, S., Scibora, L., and Bardy, B. G. (2010). Stance width influences postural stability and motion sickness. Ecol. Psychol. 22, 169-191. doi: 10.1080/10407413.2010.496645

St. Pierre, M. E., Banerjee, S., Hoover, A. W., and Muth, E. R. (2015). The effects of $0.2 \mathrm{~Hz}$ varying latency with $20-100 \mathrm{~ms}$ varying amplitude on simulator sickness in a helmet mounted display. Displays 36, 1-8. doi: 10.1016/j.displa.2014.10.005

Tanahashi, S., Ujike, H., Kozawa, R., and Ukai, K. (2007). Effects of visually simulated roll motion on vection and postural stabilization. J. NeuroEng. Rehabil. 4:39. doi: 10.1186/1743-0003-4-39

Tate, D. L., Sibert, L., and King, T. (1997). "Virtual environments for shipboard firefighting training," in Proceedings of IEEE 1997 Annual International Symposium on Virtual Reality (Albuquerque, NM: IEEE), 61-68. doi: 10.1109/VRAIS.1997.583045

Teixeira, J., and Palmisano, S. (2020). The effect of dynamic field-of-view restriction on experiences of cybersickness and presence in HMD-based virtual reality. Virtual Real. doi: 10.1007/s10055-020-00466-2

Thornton, W. E., and Bonato, F. (2013). Space motion sickness and motion sickness: symptoms and etiology. Aviat. Space Environ. Med. 84, 716-721. doi: 10.3357/ASEM.3449.2013

Treisman, M. (1977). Motion sickness: an evolutionary hypothesis. Science 197, 493-495. doi: 10.1126/science.301659

Valko, Y., Lewis, R. F., Priesol, A. J., and Merfeld, D. M. (2012). Vestibular labyrinth contributions to human whole-body motion discrimination. J. Neurosci. 32, 13537-13542. doi: 10.1523/JNEUROSCI.2157-12.2012

Van Waveren, J. M. P. (2016). "The asynchronous time warp for virtual reality on consumer hardware," in Proceedings of the 22nd ACM Conference on Virtual Reality Software and Technology (Munich: ACM), 37-46. doi: $10.1145 / 2993369.2993375$

Villani, D., Riva, F., and Riva, G. (2007). New technologies for relaxation: the role of presence. Int. J. Stress Manage. 14, 260-274. doi: 10.1037/1072-5245.14.3.260

Villard, S. J., Flanagan, M. B., Albanese, G. M., and Stoffregen, T. A. (2008). Postural instability and motion sickness in a virtual moving room. Hum. Factors 50, 332-345. doi: 10.1518/001872008X250728
Warwick-Evans, L. A., Symons, N., Fitch, T., and Burrows, L. (1998). Evaluating sensory conflict and postural instability. Theories of motion sickness. Brain Res. Bull. 47, 465-469. doi: 10.1016/S0361-9230(98)00090-2

Webb, N. A., and Griffin, M. J. (2002). Optokinetic stimuli: motion sickness, visual acuity, and eye movements. Aviat. Space Environ. Med. 73, 351-358.

Webb, N. A., and Griffin, M. J. (2003). Eye movement, vection, and motion sickness with foveal and peripheral vision. Aviat. Space Environ. Med. $74,622-625$.

Weech, S., Varghese, J. P., and Barnett-Cowan, M. (2018). Estimating the sensorimotor components of cybersickness. J Neurophysiol. 120, 2201-2217. doi: 10.1152/jn.00477.2018

Wiederhold, B. K., Gao, K., Sulea, C., and Wiederhold, M. D. (2014). Virtual reality as a distraction technique in chronic pain patients. Cyberpsychol. Behav. Soc. Netw. 17, 346-352. doi: 10.1089/cyber.2014.0207

Wu, W., Dong, Y., and Hoover, A. (2016). Measuring digital system latency from sensing to actuation at continuous 1-ms resolution. Presence 22, 20-35. doi: 10.1162/PRES_a_00131

Yildirim, C. (2019a). Don't make me sick: investigating the incidence of cybersickness in commercial virtual reality headsets. Virtual Real. 24, 1-9. doi: 10.1007/s10055-019-00401-0

Yildirim, C. (2019b). Cybersickness during VR gaming undermines game enjoyment: A mediation model. Displays 59, 35-43. doi: 10.1016/j.displa.2019.07.002

Yokokohji, Y., Sugawara, Y., and Yoshikawa, T. (2000). "Accurate image overlay on video see-through HMDs using vision and accelerometers," in Proceedings of IEEE Virtual Reality (New Brunswick, NJ: IEEE), 247-254. doi: 10.1109/VR.2000.840505

Yokota, Y., Aoki, M., Mizuta, K., Ito, Y., and Isu, N. (2005). Motion sickness susceptibility associated with visually induced postural instability and cardiac autonomic responses in healthy subjects. Acta Otolaryngol. 125, 280-285. doi: 10.1080/00016480510003192

Zhao, J., Allison, R. S., Vinnikov, M., and Jennings, S. (2017). "Estimating the motion-to-photon latency in head mounted displays," in Proceedings of IEEE Virtual Reality (Los Angeles, CA: IEEE), 313-314. doi: 10.1109/VR.2017.7892302

Conflict of Interest: The authors declare that the research was conducted in the absence of any commercial or financial relationships that could be construed as a potential conflict of interest.

Copyright (c) 2020 Palmisano, Allison and Kim. This is an open-access article distributed under the terms of the Creative Commons Attribution License (CC BY). The use, distribution or reproduction in other forums is permitted, provided the original author(s) and the copyright owner(s) are credited and that the original publication in this journal is cited, in accordance with accepted academic practice. No use, distribution or reproduction is permitted which does not comply with these terms. 